\title{
Fast Computation of Convolution Operations via Low-Rank Approximation
}

\author{
Tristan A. Hearn* Lothar Reichel ${ }^{\dagger}$
}

\begin{abstract}
Methods for the approximation of 2D discrete convolution operations are derived for the case when a low-rank approximation of one of the input matrices is available. Algorithms based on explicit computation and on the Fast Fourier Transform are described. Applications to the computation of cross-correlation and autocorrelation are discussed. Both theory and numerical experiments show that the use of low-rank approximations makes it possible to determine accurate approximations of convolutive operations at competitive speed.
\end{abstract}

\section{Introduction}

Discrete convolution is a fundamental operation in digital signal processing. The entries of the 2D convolution $G=\left[G_{i, j}\right]$ of two matrices $F=\left[F_{k, l}\right]$ and $K=\left[K_{m, n}\right]$ are given by

$$
G_{i, j}=[F * K]_{i, j}=\sum_{h} \sum_{l} F_{h, l} K_{i-h, j-l},
$$

where $*$ denotes the discrete convolution operator. In this paper, we assume the matrices $F$ and $K$ to be real; however, this restriction easily can be removed. If the matrices $F, K$, and their convolution $G$ have the same dimensions, then boundary conditions must be specified so that the components in equation (1) are well defined also when the indices $h, l, i-h$, or $j-l$ exceed the dimensions of $F$ or $K$. If periodic boundary conditions are assumed, then the discrete convolution may be evaluated with the aid of the Fast Fourier Transform (FFT) method. By the Fourier convolution theorem, the 2D discrete Fourier transform of $F * K$ may be computed as

$$
\widehat{F * K}=\hat{F} \cdot \hat{K},
$$

*Department of Mathematical Sciences, Kent State University, Kent, OH 44242, USA. E-mail: tristan.a.hearn@nasa.gov.

${ }^{\dagger}$ Department of Mathematical Sciences, Kent State University, Kent, OH 44242, USA. E-mail: reichel@math.kent.edu. Research supported in part by NSF grant DMS1115385 . 
where $\cdot$ denotes component-wise multiplication, and $\hat{F}$ and $\hat{K}$ are the 2D discrete Fourier transforms of $F$ and $K$, respectively. FFT-based computations of convolution operations may be much faster than explicit computation of these operations; however, the computing time depends both on the dimensions of the input data and on the need for preprocessing (such as zeropadding); see [11]. We will discuss both explicit and FFT-based approaches to computing convolution operations.

Convolution is a tool with a wide variety of applications in applied mathematics [6, 10], statistics [9], physics [31, 32], image processing and engineering $[20,23,24,28]$, as well as in other disciplines [7, 26]. This paper is concerned with convolution in two space-dimensions and relevant applications, principally applications to digital image processing. For example, edge detection within an image can be performed by convolving the matrix representing the pixels of the image with another matrix whose entries represent the stencil of a discretized differential operator. Another application is the "matched filtering" method for object detection in a digital image. This method computes the convolution of the matrix representing the image with a mirror-image of the matrix representing an image of the object to be detected; see [14]. This operation also is known as cross-correlation, and is discussed in the following section.

Discrete convolutions are computationally demanding operations. Many approaches have been suggested for speeding up their computation. For instance, the convolution operation can be decomposed into parallelizable operations that can be executed efficiently on parallel computing hardware $[1,33]$ or on computer graphics processors [18]. This paper introduces new methods for computing 2D discrete convolutions in situations when low-rank approximations of one or both of the matrices to be convolved are available. The proposed methods are suitable both when the convolution is computed explicitly by (1) or by using the FFT, and they are well suited for implementation on parallel computing architectures. It is the purpose of this paper to illustrate that in many applications that require the computation of convolutions with a matrix $K$, such as when computing auto- or crosscorrelations, it may suffice to approximate $K$ by a matrix of very low rank. It is then possible to evaluate the convolution explicitly in an efficient manner. Computed examples show that this approach can be both faster and more accurate than the standard approach based on applying the FFT. The higher accuracy stems from that the desired convolution is not approximated by a cyclic one. We believe the observation that low-rank approximations give sufficiently accurate results and fast numerical methods to be new.

This paper is organized as follows. The cross-correlation and autocorrelation operations, as well as FFT-based computations of convolutions, are introduced in Section 2. Evaluation of convolutive operations when one of the matrices involved is of low rank is described in Section 3. Error bounds for approximations of the convolutive operations when the low-rank approximation is a truncated singular value decomposition are derived in Section 4, 
and numerical examples are presented in Section 5. Concluding remarks can be found in Section 6 .

\section{Convolution-based operations}

An immediate application of the convolution is the evaluation of the crosscorrelation $C=\left[C_{i, j}\right]$ of two matrices $F=\left[F_{h, l}\right]$ and $K=\left[K_{m, n}\right]$. It is defined by

$$
C_{i, j}=\sum_{h} \sum_{l} F_{h, l} K_{i+h, j+l}
$$

and can be written as the convolution $C=F * \overleftarrow{K}$, where $\overleftarrow{K}$ denotes the matrix obtained by reversing each column and each row of the matrix $K$. It follows that any advancement in efficient computation of convolutions may immediately be applied to the computation of cross-correlations. Beyond the already mentioned application to matched filtering, cross-correlation is a component of more sophisticated analysis and detection methods for digital images and other data structures [5].

The discrete autocorrelation operation on a matrix $F$ also can be expressed as a convolution operation, because the autocorrelation of $F$ is the cross-correlation of $F$ with itself. Thus, the components of the autocorrelation $H=\left[H_{i, j}\right]$ of a matrix $F=\left[F_{k, l}\right]$ can be computed as

$$
H_{i, j}=[F * \stackrel{\leftarrow}{F}]_{i, j}=\sum_{h} \sum_{l} F_{h, l} F_{i+h, j+l}
$$

We note that the Fourier convolution theorem makes it possible to compute the autocorrelation in the frequency domain:

$$
\widehat{H}=\widehat{F * \overleftarrow{F}}=\widehat{F} \cdot \widehat{\overleftarrow{F}}
$$

Autocorrelation is a standard tool in the analysis of digital signals. For example, it is used in audio engineering for pitch detection [25] and estimation of optical spectra of laser light [13]. Moreover, it is used for the analysis of digital images; autocorrelation plays a role in methods for estimating the spatial distribution of recurring features within images [12], and as a component in recently developed autofocus methods in digital image deblurring $[29,30]$.

\section{Derivation of the methods}

Our methods for approximating and evaluating convolution operators use the separability and linearity of the convolution. This section discusses the computation of the convolution of a matrix $F$ with a convolution kernel of 
low rank. No low-rank approximation of $F$ is required. However, for the approximation of the autocorrelation $F * \stackrel{\leftarrow}{F}$ a low-rank approximation of the matrix $F$ is assumed to be known. Error bounds when the low-rank approximations are determined from a partial singular value decomposition are discussed in Section 4.

Let $K_{j}$ and $F_{j}$ be rank- $j$ approximations of $K$ and $F$, respectively. These approximations can be written as sums of $j$ rank-1 matrices. For example, we can express $K_{j} \in \mathbb{R}^{m \times n}$ as

$$
K_{j}=\sum_{i=1}^{j} \gamma_{i} u_{i} v_{i}^{T}
$$

for some $\gamma_{i} \in \mathbb{R}$ and vectors $u_{i} \in \mathbb{R}^{m}$ and $v_{i} \in \mathbb{R}^{n}$ with $\left\|u_{i}\right\|_{2}=\left\|v_{i}\right\|_{2}=1$. Throughout this paper $\|\cdot\|_{2}$ refers to the Euclidean vector norm or the associated induced matrix norm. The superscript ${ }^{T}$ in (6) denotes transposition.

The singular value decomposition (SVD) of $K$ can be used to determine an optimal approximation of rank $j$ with respect to the spectral norm and the Frobenius norm. Also the framework of Independent Component Analysis (ICA) [21] can be applied to compute a rank- $j$ approximation that is optimal in some sense; specifically, the approximation is optimal with respect to a high order measure of statistical independence. Since the error in low-rank approximants determined by the SVD can be determined quite easily, while this is not the case for low-rank approximants computed by using ICA, we focus in this paper on the former.

The following assumptions on the dimensions of the matrices $F$ and $K$ are made. The discussion in Section 3.1.1 on explicit convolution of the matrices $F$ and $K$ does not require these matrices to be of particular dimensions. However, the description in Section 3.1.2 of FFT-based convolution demands the matrices $F$ and $K$ to be of equal dimensions. This can be achieved by zero-padding.

\subsection{The convolution $F * K_{j}$}

We first consider the convolution of a matrix $F \in \mathbb{R}^{m_{1} \times n_{1}}$ with a low-rank approximation $K_{j} \in \mathbb{R}^{m_{2} \times n_{2}}$ of a kernel $K \in \mathbb{R}^{m_{2} \times n_{2}}$. The convolution with $K_{j}$ is a linear operation on $F$. It can be written as

$$
\operatorname{vec}(G)=A \operatorname{vec}(F),
$$

where $A$ is a matrix that encodes the discrete convolution operation with $K_{j}$, and $\operatorname{vec}(G)$ and $\operatorname{vec}(F)$ are column vectors formed by successively stacking the columns of $G=\left[g_{1}, g_{2}, \ldots, g_{n_{1}}\right]=\left[g_{i, j}\right]$ and $F=\left[f_{1}, f_{2}, \ldots, f_{n_{1}}\right]=\left[f_{i, j}\right]$, 
i.e.,

$$
\operatorname{vec}(G)=\left[\begin{array}{c}
g_{1} \\
g_{2} \\
\vdots \\
g_{n_{1}}
\end{array}\right]=\left[\begin{array}{c}
g_{1,1} \\
\vdots \\
g_{m_{1}, 1} \\
\vdots \\
g_{m_{1}, n_{1}}
\end{array}\right], \quad \operatorname{vec}(F)=\left[\begin{array}{c}
f_{1} \\
f_{2} \\
\vdots \\
f_{n_{1}}
\end{array}\right]=\left[\begin{array}{c}
f_{1,1} \\
\vdots \\
f_{m_{1}, 1} \\
\vdots \\
f_{m_{1}, n_{1}}
\end{array}\right] .
$$

We describe algorithms for explicit and FFT-based evaluation of convolutions separately.

\subsubsection{Explicit computation}

It follows from (1) that the computation of one component of the convolution $F * K$ requires $m_{2} n_{2}$ multiplications. Using this formula to compute all entries of $F * K$ requires $m_{2} n_{2} m_{1} n_{1}$ multiplications. The following result, discussed in $[16,22]$, shows how the computations can be simplified when $K$ is a rankone matrix.

Lemma 3.1. Let $F \in \mathbb{R}^{m \times n}, u \in \mathbb{R}^{m}, v \in \mathbb{R}^{n}$, and $K_{1}=u v^{T}$. Then

$$
\operatorname{vec}\left(F * K_{1}\right)=\left(A_{r} \otimes A_{c}\right) \operatorname{vec}(F)
$$

for some matrices $A_{r} \in \mathbb{R}^{n \times n}$ and $A_{c} \in \mathbb{R}^{m \times m}$, where $\otimes$ denotes the Kronecker product

$$
A_{r} \otimes A_{c}=\left[\begin{array}{ccc}
a_{r_{1,1}} a_{c_{1,1}} & \cdots & a_{r_{1, n}} a_{c_{1, m}} \\
\vdots & \ddots & \vdots \\
a_{r_{n, 1}} a_{c_{m, 1}} & \cdots & a_{r_{n, n}} a_{c_{m, m}}
\end{array}\right]
$$

The matrices $A_{r}$ and $A_{c}$ in Lemma 3.1 perform discrete 1D convolutions with $u$ on the columns of $F$ and with $v$ on the rows of $F$, respectively; see [16]. Hence, the computation of a single component of $F * K_{1}$ degenerates to the computation of two 1D discrete convolutions. Together, their evaluation requires only $m_{2}+n_{2}$ multiplications. Taken over all components of $F$, the full computation of $F * K_{1}$ requires only $\left(m_{2}+n_{2}\right) m_{1} n_{1}$ multiplications. We now consider the case when $F$ is convolved with a rank- $j$ matrix.

Theorem 3.2. Let $F \in \mathbb{R}^{m \times n}$ and $K_{j}=\sum_{i=1}^{j} \gamma_{i} u_{i} v_{i}^{T} \in \mathbb{R}^{m \times n}$. Then, for some matrices $A_{r_{i}} \in \mathbb{R}^{n \times n}$ and $A_{c_{i}} \in \mathbb{R}^{m \times m}, i=1,2, \ldots, j$, we have

$$
\operatorname{vec}\left(F * K_{j}\right)=\left[\sum_{i=1}^{j} \gamma_{i}\left(A_{r_{i}} \otimes A_{c_{i}}\right)\right] \operatorname{vec}(F) \text {. }
$$


Proof. The linearity of the convolution operation yields

$$
F * K_{j}=F * \sum_{i=1}^{j} \gamma_{i} u_{i} v_{i}^{T}=\sum_{i=1}^{j}\left[\gamma_{i} F *\left(u_{i} v_{i}^{T}\right)\right]
$$

and by Lemma 3.1 each term gives

$$
\operatorname{vec}\left(\gamma_{i} F *\left(u_{i} v_{i}^{T}\right)\right)=\gamma_{i}\left(A_{r_{i}} \otimes A_{c_{i}}\right) \operatorname{vec}(F) .
$$

Therefore, we can express the discrete convolution operation as the matrixvector product

$$
\operatorname{vec}\left(F * K_{j}\right)=\sum_{i=1}^{j}\left[\gamma_{i}\left(A_{r_{i}} \otimes A_{c_{i}}\right) \operatorname{vec}(F)\right]=\left[\sum_{i=1}^{j} \gamma_{i}\left(A_{r_{i}} \otimes A_{c_{i}}\right)\right] \operatorname{vec}(F) .
$$

Theorem 3.2 shows that the convolution $F * K_{j}$ can be written as a weighted sum of convolutions of $F$ with rank-one matrices. Using this representation, the evaluation of $F * K_{j}$ requires $j\left(m_{2}+n_{2}+1\right) m_{1} n_{1}$ multiplications. Let $K_{j}$ be a rank- $j$ approximation of the matrix $K$ with $j<\frac{m_{1} n_{1}}{m_{1}+n_{1}}$. Then the evaluation of $F * K_{j}$ by the method of Theorem 3.2 requires fewer multiplications than the direct computation of $F * K$ by (1). This result forms the basis for the methods proposed in the present paper. The evaluation of $F * K_{j}$ is summarized in Algorithm 1 .

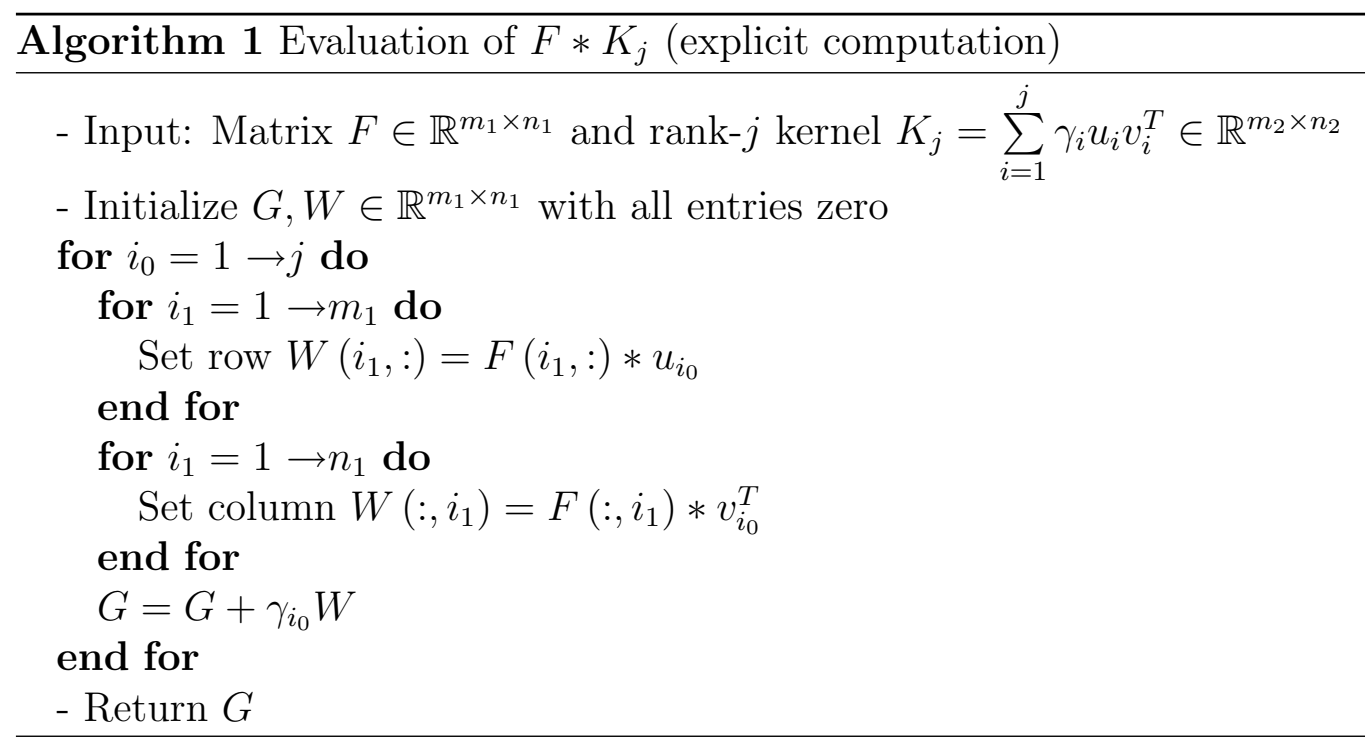

\subsubsection{FFT-based computations}

We consider the situation when the convolution is evaluated with the aid of the FFT, rather than by explicit computation. Here we assume that both $F$ 
and $K$ have the same dimensions, i.e., $F, K \in \mathbb{R}^{m \times n}$. The matrix $K$ may be zero-padded to achieve this. Let $\widehat{x}$ denote the $1 \mathrm{D}$ discrete Fourier transform of a vector $x \in \mathbb{R}^{n}$ and let $\hat{A}$ be the $2 \mathrm{D}$ discrete Fourier transform of the matrix $A \in \mathbb{R}^{m \times n}$. The latter can be computed, e.g., by applying $1 \mathrm{D}$ discrete Fourier transforms to each row and each column of $A$. We assume that 2D discrete Fourier transforms are computed in this manner in the multiplication counts reported below.

We first compare the number of 1D discrete Fourier transforms that have to be computed when evaluating $F * K$ and $F * K_{j}$, where $K_{j}$ is a kernel of rank $j$ that approximates $K$. By the Fourier convolution theorem (2), the 2D discrete Fourier transform of $F * K$ may be computed by component-wise multiplication. This requires the computation of $(m+n)+(m+n)+(m+n)=$ $3(m+n)$ separate 1D Fourier transforms (including the computation of the needed inverse discrete Fourier transforms).

Theorem 3.3. Let $F \in \mathbb{R}^{m \times n}$ and $K_{j}=\sum_{i=1}^{j} \gamma_{i} u_{i} v_{i}^{T} \in \mathbb{R}^{m \times n}$. Then the discrete Fourier transform of the discrete convolution operation $F * K_{j}$ can be evaluated as

$$
\widehat{F * K}_{j}=\sum_{h=1}^{j} \gamma_{h} \widehat{F} \cdot \widehat{u}_{h} \widehat{v}_{h}^{T}
$$

Proof. By the linearity of the Fourier transform, the 2D discrete Fourier transform of $F * K_{j}$ may be computed as

$$
\begin{aligned}
\widehat{F * K_{j}} & =\widehat{F} \cdot \widehat{K_{j}}=\widehat{F} \cdot\left[\sum_{h=1}^{j} \gamma_{h} u_{h} v_{h}^{T}\right] \\
& =\sum_{h=1}^{j} \gamma_{h} \widehat{F} \cdot \widehat{u_{h} v_{h}^{T}}=\sum_{h=1}^{j} \gamma_{h} \widehat{F} \cdot \widehat{u}_{h} \widehat{v}_{h}^{T} .
\end{aligned}
$$

The computations of Theorem 3.3 require only the evaluation of $(m+n)+$ $2 j+(m+n)=2(m+n)+2 j 1 \mathrm{D}$ discrete Fourier transforms. Thus, if $j<\frac{m+n}{2}$, then $F * K_{j}$ may be computed with fewer applications of 1D discrete Fourier transforms by using the structure of $K_{j}$ than by considering $K_{j}$ an arbitrary matrix in $\mathbb{R}^{m \times n}$. Algorithm 2 summarizes the computations of Theorem 3.3.

\subsection{The autocorrelation of $F$}

\subsubsection{Explicit computation}

Similarly as above, we let $\overleftarrow{F}$ denote the matrix obtained by reversing each column and each row of $F$. Then the autocorrelation of $F$ can be computed 


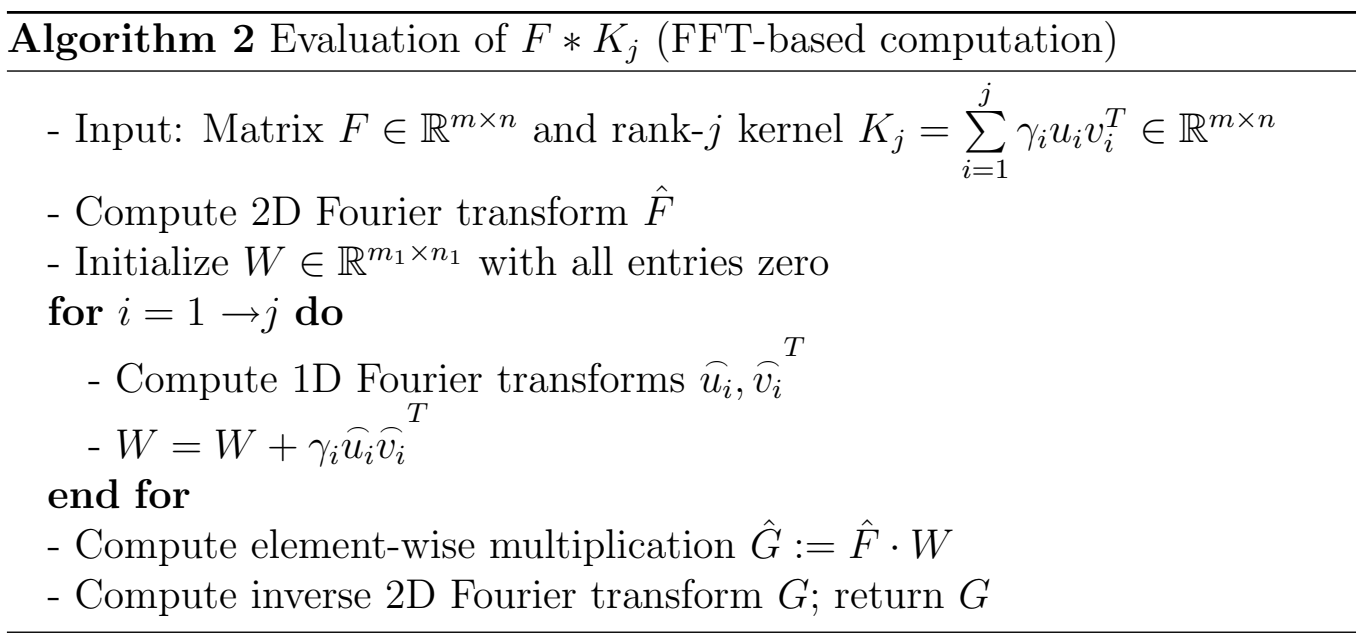

as the convolution $F * \overleftarrow{F}$. An explicit computation of the autocorrelation of $F$ requires $m^{2} n^{2}$ multiplications, as discussed in Section 3.1.1. This section is concerned with the exploitation of the structure when $F$ is a matrix of low rank. We first consider the computation of the convolution of two rank-one matrices.

Lemma 3.4. Let $u_{1}, u_{2} \in \mathbb{R}^{m}, v_{1}, v_{2} \in \mathbb{R}^{n}$, and $\gamma_{1}, \gamma_{2} \in \mathbb{R}$. Then

$$
\left(\gamma_{1} u_{1} v_{1}^{T}\right) *\left(\gamma_{2} u_{2} v_{2}^{T}\right)=\gamma_{1} \gamma_{2}\left(u_{1} * u_{2}\right)\left(v_{1} * v_{2}\right)^{T}
$$

Proof. By linearity, we have

$$
\left(\gamma_{1} u_{1} v_{1}^{T}\right) *\left(\gamma_{2} u_{2} v_{2}^{T}\right)=\gamma_{1} \gamma_{2}\left(u_{1} v_{1}^{T}\right) *\left(u_{2} v_{2}^{T}\right)
$$

From the definition $(1)$, the $(i, j)$ th component of the above convolution is given by

$$
\gamma_{1} \gamma_{2} \sum_{h=1}^{m} \sum_{l=1}^{n}\left(u_{1} v_{1}^{T}\right)_{i-h, j-l}\left(u_{2} v_{2}^{T}\right)_{h, l} .
$$

Using that $\left[u v^{T}\right]_{i, j}=u_{i} v_{j}^{T}$ for any outer product $u v^{T}$, the above equation can be written as

$$
\begin{aligned}
\gamma_{1} \gamma_{2} \sum_{h=1}^{m} \sum_{l=1}^{n} u_{1_{i-h}} v_{1_{j-l}}^{T} u_{2_{h}} v_{2_{l}}^{T} & =\gamma_{1} \gamma_{2} \sum_{h=1}^{m} \sum_{l=1}^{n} u_{1_{i-h}} u_{2_{h}} v_{1_{j-l}}^{T} v_{2_{l}}^{T} \\
& =\gamma_{1} \gamma_{2} \sum_{h=1}^{m} u_{1_{i-h}} u_{2_{h}} \sum_{l=1}^{n} v_{1_{j-l}}^{T} v_{2_{l}}^{T},
\end{aligned}
$$

where the last equality follows by changing the order of summation. The sum $(7)$ is recognized as the $(i, j)$ th component of the outer product of the two 1D convolutions

$$
\gamma_{1} \gamma_{2}\left(u_{1} * u_{2}\right)\left(v_{1} * v_{2}\right)^{T}
$$


We apply the above result to the evaluation of the autocorrelation of a rank- $j$ matrix.

Theorem 3.5. Let $F_{j}=\sum_{i=1}^{j} \gamma_{i} u_{i} v_{i}^{T} \in \mathbb{R}^{m \times n}$. Then the autocorrelation $F_{j} * \overleftarrow{F}_{j}$ can be computed as

$$
F_{j} * \overleftarrow{F}_{j}=\sum_{i=1}^{j} \gamma_{i}^{2}\left(u_{i} * \overleftarrow{u}_{i}\right)\left(v_{i} * \overleftarrow{v}_{i}\right)^{T}+2 \sum_{i=1}^{j} \sum_{h>i}^{j} \gamma_{i} \gamma_{h}\left(u_{i} * \overleftarrow{u}_{h}\right)\left(v_{i} * \overleftarrow{v}_{h}\right)^{T}
$$

Proof. Direct expansion gives

$$
\begin{aligned}
F_{j} * \overleftarrow{F}_{j} & =\left(\sum_{i=1}^{j} \gamma_{i} u_{i} v_{i}^{T}\right) * \overleftarrow{\left(\sum_{h=1}^{j} \gamma_{h} u_{h} v_{h}^{T}\right)} \\
& =\left(\sum_{i=1}^{j} \gamma_{i} u_{i} v_{i}^{T}\right) *\left(\sum_{h=1}^{j} \gamma_{h} \overleftarrow{u}_{h} \overleftarrow{v}_{h}^{T}\right) \\
& =\sum_{i=1}^{j}\left(\left(\gamma_{i} u_{i} v_{i}^{T}\right) * \sum_{h=1}^{j} \gamma_{h} \overleftarrow{u}_{h} \overleftarrow{v}_{h}^{T}\right) \\
& =\sum_{i=1}^{j} \sum_{h=1}^{j} \gamma_{i} \gamma_{h}\left(u_{i} v_{i}^{T}\right) *\left(\overleftarrow{u}_{h} \overleftarrow{v}_{h}^{T}\right) .
\end{aligned}
$$

By Lemma 3.4 this is equivalent to

$$
\begin{aligned}
& \sum_{i=1}^{j} \sum_{h=1}^{j} \gamma_{i} \gamma_{h}\left(u_{i} v_{i}^{T}\right) *\left(\overleftarrow{u}_{h} \overleftarrow{v}_{h}^{T}\right)=\sum_{i=1}^{j} \sum_{h=1}^{j} \gamma_{i} \gamma_{h}\left(u_{i} * \overleftarrow{u}_{h}\right)\left(v_{i} * \overleftarrow{v}_{h}\right)^{T} \\
& =\sum_{i=1}^{j} \gamma_{i}^{2}\left(u_{i} * \overleftarrow{u}_{i}\right)\left(v_{i} * \overleftarrow{v}_{i}\right)^{T}+2 \sum_{i=1}^{j} \sum_{h>i}^{j} \gamma_{i} \gamma_{h}\left(u_{i} * \overleftarrow{u}_{h}\right)\left(v_{i} * \overleftarrow{v}_{h}\right)^{T} .
\end{aligned}
$$

Performing the computations of Theorem 3.5 requires

$$
j(1+m n+m n)+2 \frac{j^{2}}{2}(1+m n+m n)=j(1+2 m n)+j^{2}(1+2 m n)
$$

multiplications. Therefore, if

$$
j<\frac{1}{2}\left(\sqrt{\frac{4 m^{2} n^{2}+2 m n+1}{2 m n+1}}-1\right),
$$

then this method of approximating the autocorrelation requires fewer multiplications than when ignoring the structure of $F_{j}$. The computations are summarized in Algorithm 3. 


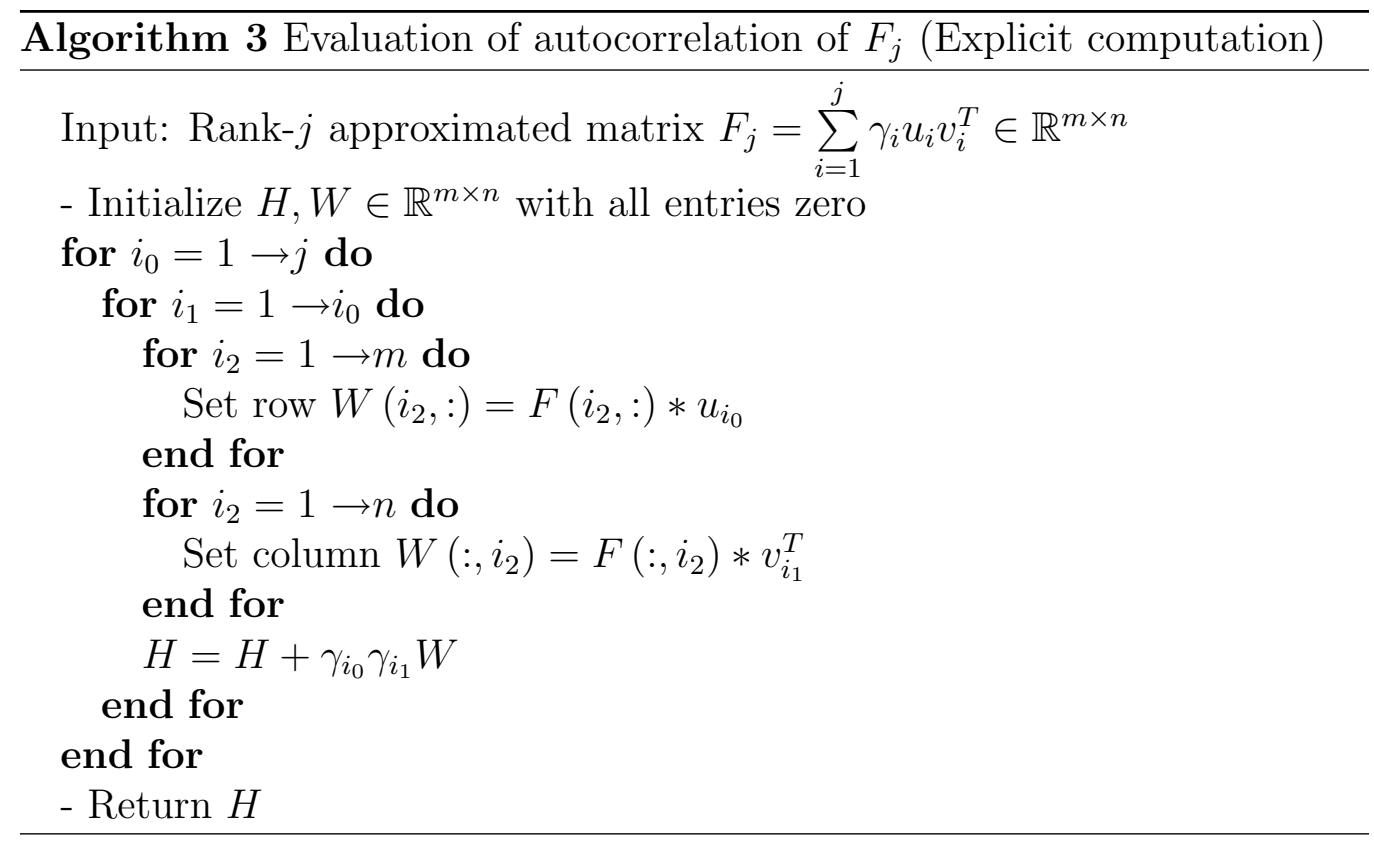

\subsubsection{FFT-based computation}

The Fourier transform of the autocorrelation of $F$ may be computed as

$$
\widehat{F * \overleftarrow{F}}=\widehat{F} \cdot \overleftrightarrow{F}=\widehat{F} \cdot \widehat{\widehat{F}}=|\widehat{F}|^{2}
$$

where $|M|^{2}$ denotes the matrix whose entries are the magnitude squared of the entries of $M$, and $\bar{M}$ denotes entry-wise complex conjugation. Thus, the autocorrelation of the matrix $F$ is the inverse 2D discrete Fourier transform of its power spectral density. The relation (8) and its application are discussed, e.g., in [8]. We describe the computation of the autocorrelation of a matrix of $\operatorname{rank} j$.

Theorem 3.6. Let $F_{j}=\sum_{i=1}^{j} \gamma_{i} u_{i} v_{i}^{T} \in \mathbb{R}^{m \times n}$. Then the Fourier transform of the autocorrelation $F_{j} * \overleftarrow{F}_{j}$ can be computed as

$$
\left|\sum_{i=1}^{j} \gamma_{i} \widehat{u}_{i} \widehat{v}_{i}^{T}\right|^{2}
$$

Proof. We obtain from (8) that

$$
\widehat{F_{j} * \overleftarrow{F}_{j}}=\left|\hat{F}_{j}\right|^{2}=\left|\widehat{\sum_{i=1}^{j} \gamma_{i} u_{i} v_{i}^{T}}\right|^{2}=\left|\sum_{i=1}^{j} \gamma_{i} \widehat{u_{i} v_{i}^{T}}\right|^{2}=\left|\sum_{i=1}^{j} \gamma_{i} \widehat{u}_{i} \widehat{v}_{i}^{T}\right|^{2} .
$$


Thus, by Theorem 3.6, we can compute $F_{j} * \overleftarrow{F}_{j}$ by evaluating only $m+$ $n+2 j 1 \mathrm{D}$ discrete Fourier transforms. If $j<\frac{m+n}{2}$, then the computation of $F_{j} * \overleftarrow{F}_{j}$ in this manner requires fewer evaluations of $1 \mathrm{D}$ discrete Fourier transform than when the structure of $F_{j}$ is ignored. The computations using the approach of Theorem 3.6 are summarized in Algorithm 4.

$\overline{\text { Algorithm } 4 \text { Evaluation of autocorrelation of } F_{j} \text { (FFT-based computation) }}$

Input: Rank- $j$ matrix $F_{j}=\sum_{i=1}^{j} \gamma_{i} u_{i} v_{i}^{T} \in \mathbb{R}^{m \times n}$

- Initialize $H \in \mathbb{R}^{m \times n}$ with all entries zero

for $i=1 \rightarrow j$ do

- Compute 1D Fourier transforms $\widehat{u_{i}}, \widehat{v_{i}}$

- $\hat{H}=\hat{H}+\gamma_{i}{\widehat{u_{i}}}^{\widehat{v}_{i}^{T}}$

end for

- Compute inverse 2D discrete Fourier transform $H$; return $H$

\section{Accuracy of low-rank approximations}

We described in the previous section how convolutive operations involving low-rank matrices can be evaluated efficiently. This section is concerned with the error in the computed result when a matrix or kernel of full rank are approximated by a truncated SVD of low rank of the matrix or kernel.

\subsection{The convolution $F * K_{j}$}

Theorem 4.1. Let $F \in \mathbb{R}^{m_{1} \times n_{1}}$ and let

$$
K_{j}=\sum_{i=1}^{j} \gamma_{i} u_{i} v_{i}^{T} \in \mathbb{R}^{m_{2} \times n_{2}}
$$

be a rank-j approximation of the matrix $K=\sum_{i=1}^{r} \gamma_{i} u_{i} v_{i}^{T} \in \mathbb{R}^{m_{2} \times n_{2}}$ of rank $r \geq j$ with the $u_{i}$ and $v_{i}$ unit vectors. Then

$$
\frac{\left\|\operatorname{vec}\left(K * F-K_{j} * F\right)\right\|_{2}}{\|\operatorname{vec}(F)\|_{2}} \leqslant \sum_{i=j+1}^{r}\left|\gamma_{i}\right| \sigma_{c_{i}} \sigma_{r_{i}},
$$

where the $\sigma_{c_{i}}$ and $\sigma_{r_{i}}$ are the largest singular values of the matrices $A_{r_{i}}$ and $A_{c_{i}}$, respectively, of the $1 D$ convolution operations constructed from the representation (9) of $K_{j}$. 
Proof. The linearity of the convolution and Lemma 3.2 give

$$
\begin{aligned}
\frac{\left\|\operatorname{vec}\left(K * F-K_{j} * F\right)\right\|_{2}}{\|\operatorname{vec}(F)\|_{2}} & =\frac{\left\|\operatorname{vec}\left(\left(K-K_{j}\right) * F\right)\right\|_{2}}{\left\|\operatorname{vec}(F)_{2}\right\|} \\
& =\frac{\left\|\operatorname{vec}\left(\left[\sum_{i=j+1}^{r} \gamma_{i} u_{i} v_{i}^{T}\right] * F\right)\right\|_{2}}{\|\operatorname{vec}(F)\|_{2}} \\
& =\frac{\left\|\sum_{i=j+1}^{r} \gamma_{i}\left(A_{r_{i}} \otimes A_{c_{i}}\right) \operatorname{vec}(F)\right\|_{2}}{\|\operatorname{vec}(F)\|_{2}} .
\end{aligned}
$$

The triangle inequality and the fact that

$$
\frac{\left\|\left(A_{c_{i}} \otimes A_{r_{i}}\right) \operatorname{vec}(F)\right\|_{2}}{\|\operatorname{vec}(F)\|_{2}} \leqslant\left\|A_{c_{i}} \otimes A_{r_{i}}\right\|_{2}, \quad i=1,2, \ldots, j,
$$

yield, in order,

$$
\begin{aligned}
\frac{\left\|\sum_{i=j+1}^{r} \gamma_{i}\left(A_{r_{i}} \otimes A_{c_{i}}\right) \operatorname{vec}(F)_{2}\right\|_{2}}{\|\operatorname{vec}(F)\|_{2}} & \leqslant \sum_{i=j+1}^{r}\left|\gamma_{i}\right| \frac{\left\|\left(A_{r_{i}} \otimes A_{c_{i}}\right) \operatorname{vec}(F)\right\|_{2}}{\|\operatorname{vec}(F)\|_{2}} \\
& \leqslant \sum_{i=j+1}^{r}\left|\gamma_{i}\right|\left\|A_{r_{i}} \otimes A_{c_{i}}\right\|_{2} .
\end{aligned}
$$

Let $U_{r_{i}} \Sigma_{r_{i}} V_{r_{i}}^{T}$ and $U_{c_{i}} \Sigma_{c_{i}} V_{c_{i}}^{T}$ be the singular value decompositions of $A_{r_{i}}$ and $A_{c_{i}}$, respectively. Then

$$
A_{r_{i}} \otimes A_{c_{i}}=\left(U_{r_{i}} \otimes U_{c_{i}}\right)\left(\Sigma_{r_{i}} \otimes \Sigma_{c_{i}}\right)\left(V_{r_{i}} \otimes V_{c_{i}}\right)^{T} ;
$$

see, e.g., [19]. Let $\sigma_{r_{i}}$ and $\sigma_{c_{i}}$ denote the largest singular values of $A_{r_{i}}$ and $A_{c_{i}}$, respectively. Then

$$
\left\|A_{r_{i}} \otimes A_{c_{i}}\right\|_{2}=\sigma_{c_{i}} \sigma_{r_{i}}
$$

and it follows that

$$
\sum_{i=j+1}^{r}\left|\gamma_{i}\right|\left\|A_{r_{i}} \otimes A_{c_{i}}\right\|_{2}=\sum_{i=j+1}^{r}\left|\gamma_{i}\right| \sigma_{c_{i}} \sigma_{r_{i}} .
$$

This shows the desired result.

\subsection{The autocorrelation $F_{j} * \bar{F}_{j}$}

To derive error bounds for the proposed method of approximating autocorrelations, first consider the following result concerning the Euclidean norm of the $1 \mathrm{D}$ convolution of two vectors. 
Lemma 4.2. Let $u, v \in \mathbb{R}^{m}$ be unit vectors. Then $\|u * v\|_{2} \leqslant \sqrt{m}$.

Proof. Young's inequality [17] and the fact that $u$ and $v$ are unit vectors yield

$$
\|u * v\|_{2} \leqslant\|u\|_{2}\|v\|_{1}=\|v\|_{1} \leqslant \sqrt{m}\|v\|_{2}=\sqrt{m} .
$$

Combining Lemmas 3.4 and 4.2 gives the following bound.

Theorem 4.3. Let $F_{j}=\sum_{i=1}^{j} \gamma_{i} u_{i} v_{i}^{T} \in \mathbb{R}^{m \times n}$ be a rank-j approximation of the matrix $F=\sum_{i=1}^{r} \gamma_{i} u_{i} v_{i}^{T} \in \mathbb{R}^{m \times n}$ of rank $r \geq j$ with the $u_{i}$ and $v_{i}$ unit vectors. Then

$$
\left\|F * \bar{F}-F_{j} * \bar{F}_{j}\right\|_{2} \leqslant 2 \sqrt{m n} \sum_{i=j+1}^{r}\left|\gamma_{i}\right| \sum_{l=1}^{r}\left|\gamma_{l}\right| .
$$

Proof. Using Lemma 3.4, the norm $\left\|F * \bar{F}-F_{j} * \bar{F}_{j}\right\|_{2}$ can be expressed as

$$
\begin{aligned}
& \left\|\left(\sum_{i=1}^{r} \gamma_{i} u_{i} v_{i}^{T}\right) *\left(\sum_{i=1}^{r} \gamma_{i} \bar{u}_{i} \bar{v}_{i}^{T}\right)-\left(\sum_{i=1}^{j} \gamma_{i} u_{i} v_{i}^{T}\right) *\left(\sum_{i=1}^{j} \gamma_{i} \bar{u}_{i} \bar{v}_{i}^{T}\right)\right\|_{2} \\
= & \left\|\sum_{i=1}^{r} \sum_{l=1}^{r} \gamma_{i} \gamma_{l}\left(u_{i} * \bar{u}_{l}\right)\left(v_{i} * \bar{v}_{l}\right)^{T}-\sum_{i=1}^{j} \sum_{l=1}^{j} \gamma_{i} \gamma_{l}\left(u_{i} * \bar{u}_{l}\right)\left(v_{i} * \bar{v}_{l}\right)^{T}\right\|_{2} \\
= & \left\|\sum_{i=1}^{r} \sum_{l=j+1}^{r} \gamma_{i} \gamma_{l}\left(u_{i} * \bar{u}_{l}\right)\left(v_{i} * \bar{v}_{l}\right)^{T}+\sum_{i=j+1}^{r} \sum_{l=1}^{j} \gamma_{i} \gamma_{l}\left(u_{i} * \bar{u}_{l}\right)\left(v_{i} * \bar{v}_{l}\right)\right\|_{2} .
\end{aligned}
$$

The triangle inequality, the fact that $\left\|u v^{T}\right\|_{2}=\|u\|_{2}\|v\|_{2}$, Lemma 4.2, and $\left\|u_{i}\right\|_{2}=\left\|v_{i}\right\|_{2}=1$ give

$$
\begin{aligned}
\left\|F * \bar{F}-F_{j} * \bar{F}_{j}\right\|_{2} \leqslant & \sum_{i=1}^{r} \sum_{l=j+1}^{r}\left|\gamma_{i} \gamma_{l}\right|\left\|\left(u_{i} * \bar{u}_{l}\right)\left(v_{i} * \bar{v}_{l}\right)^{T}\right\|_{2} \\
& +\sum_{i=j+1}^{r} \sum_{l=1}^{j}\left|\gamma_{i} \gamma_{l}\right| \mid\left(u_{i} * \bar{u}_{l}\right)\left(v_{i} * \bar{v}_{l}\right)^{T} \|_{2} \\
= & \sqrt{m n}\left(\sum_{i=1}^{r} \sum_{l=j+1}^{r}\left|\gamma_{i} \gamma_{l}\right|+\sum_{i=j+1}^{r} \sum_{l=1}^{j}\left|\gamma_{i} \gamma_{l}\right|\right) \\
\leqslant & 2 \sqrt{m n} \sum_{i=j+1}^{r}\left|\gamma_{i}\right| \sum_{l=1}^{r}\left|\gamma_{l}\right| .
\end{aligned}
$$




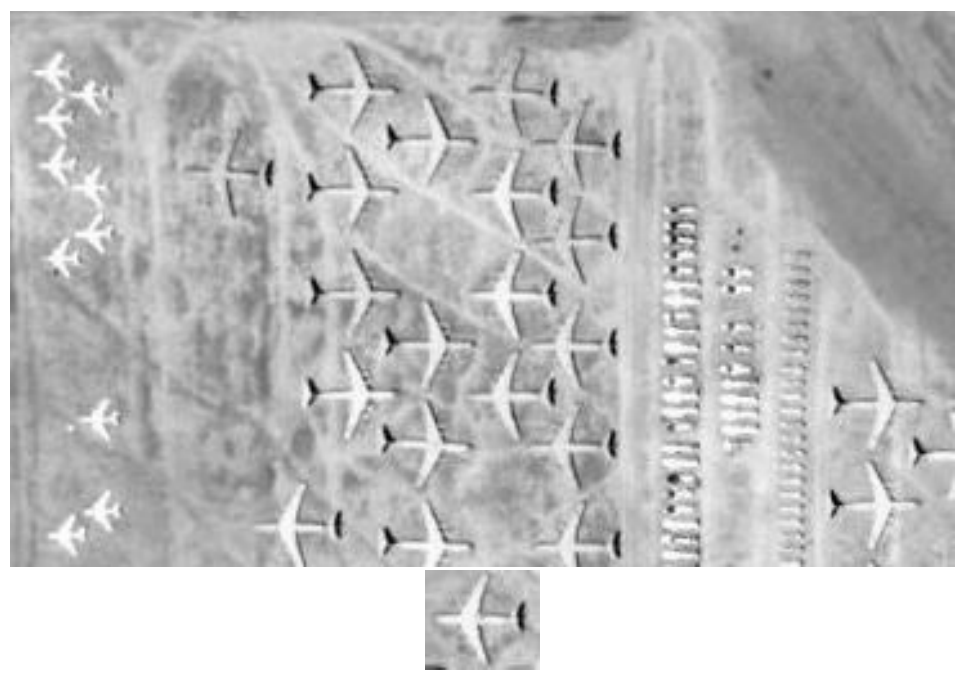

Figure 1: The original Planes and Plane images.

\section{$5 \quad$ Numerical experiments}

This section presents a few numerical examples with timings and approximation errors. All examples have been computed with code implemented in the Python ${ }^{1}$ programming language using the packages Numpy ${ }^{2}, \mathrm{Scipy}^{3}$, and Matplotlib ${ }^{4}$. The computations have been carried out on a workstation equipped with an Intel@ Core $^{\mathrm{TM}_{2}}$ Quad $2.83 \mathrm{GHz}$ CPU processor with $4 \mathrm{~GB}$ of RAM, running Linux Kernel 2.6.38-12-generic.

In the examples that use a low-rank matrix approximation, we determined this approximation with the SVD. The computational running times tabulated do not include the time needed to generate these low-rank approximations. Our timings address the situation when the same low-rank approximation is to be used many times. There are methods for the efficient computation of a partial singular value decomposition of a large matrix such as the MATLAB function svds and the schemes described in $[2,3]$. An examination of the computational efficiency of the methods discussed in this paper in combination with schemes for determining partial singular value decompositions is worthy of further study, in particular in the context of specific applications.

\subsection{Convolution with a fixed kernel}

We chose matrices that represent two gray-scale digital images, Planes and Cell, for the numerical experiments. The Planes image has dimensions $367 \times 216$, and the Cell image has dimensions $343 \times 275$. Matrices of two

\footnotetext{
${ }^{1}$ www.python.org

${ }^{2}$ www.numpy.scipy.org

${ }^{3}$ www.scipy.org

${ }^{4}$ www.matplotlib.sourceforge.net
} 


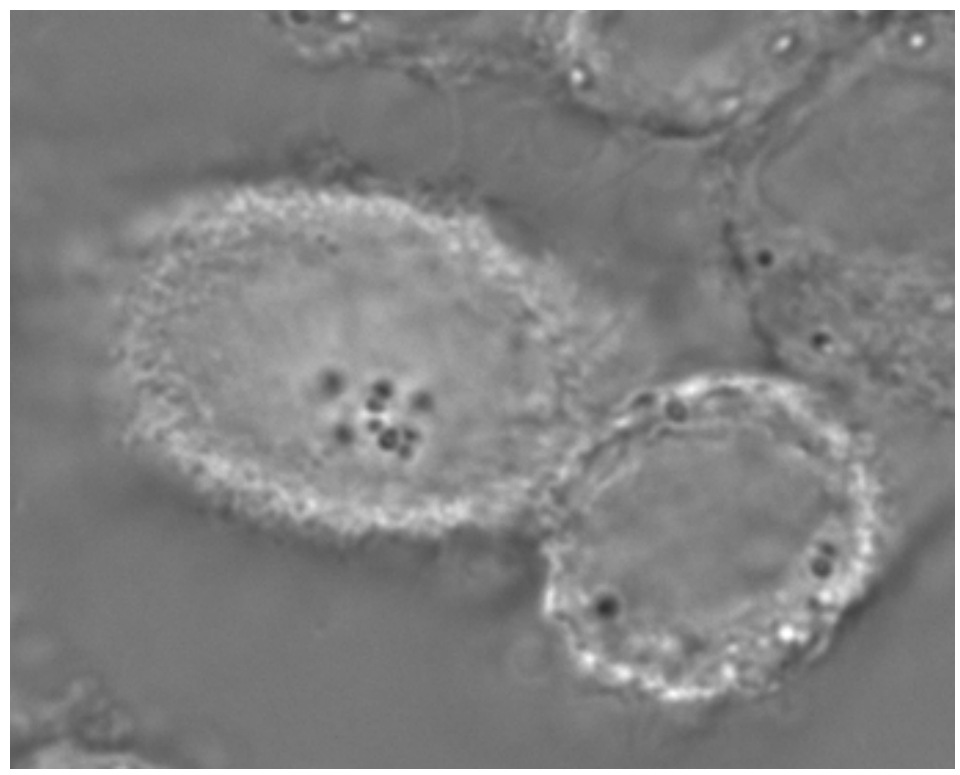

Figure 2: The original Cell and Spot images.

smaller gray-scale images, Plane and Spot were paired with Planes and Cell, respectively, to serve as convolution kernels. The Plane image has dimensions $44 \times 38$, and the Spot image has dimensions $11 \times 11$. Figure 1 shows the Planes and Plane images and Figure 2 displays the images Cell and Spot. By computing the cross-correlation between the matrix of each source image and their paired kernel, instances of features within the image which are representative of the kernel are localized through the identification of localized peaks within the components of the computed cross-correlation matrices. This application of cross-correlation operation often is referred to as "matched filtering."

We first computed the cross-correlation between each kernel-image as specified in (3), without application of the FFT. The cross-correlation matrices computed are shown in Figures 3 and 4 . Note the localized peaks in each of the cross-correlation matrices. Their position is indicative of portions of the original images that are similar in nature to the kernel used.

Next, several approximations of the cross-correlation matrices were computed using Algorithm 1 (explicit convolution). For the cross-correlation with the Planes image, rank-1,2,3,4,5,10,20, 30 approximations of the Plane image were used. The resulting approximations are shown in Figure 5. Similarly, the cross-correlation between the Cell and Spot images was approximated with rank-1,2,3,4,5,6,8,11 approximations of the Spot image, with results shown in Figure 6. Numerical data associated with these computations, averaged over five runs, are displayed in Tables 1 and 2 .

It is notable that the cross-correlations computed from a rank-2 approximation of the kernel images were sufficient for detection. Using the rank-2 


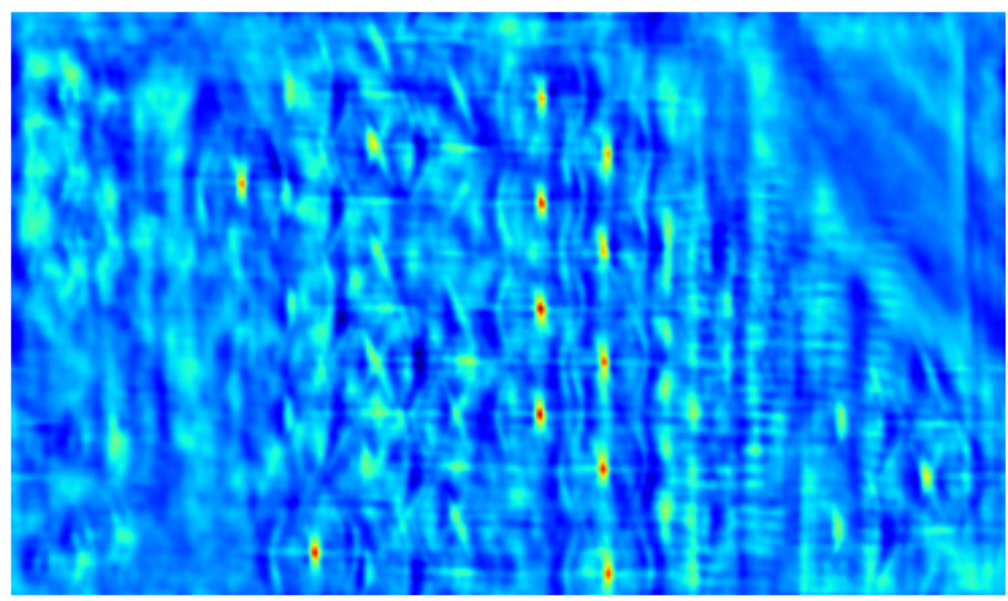

Figure 3: The computed cross-correlation of the Planes and Plane images, computed via explicit convolution.

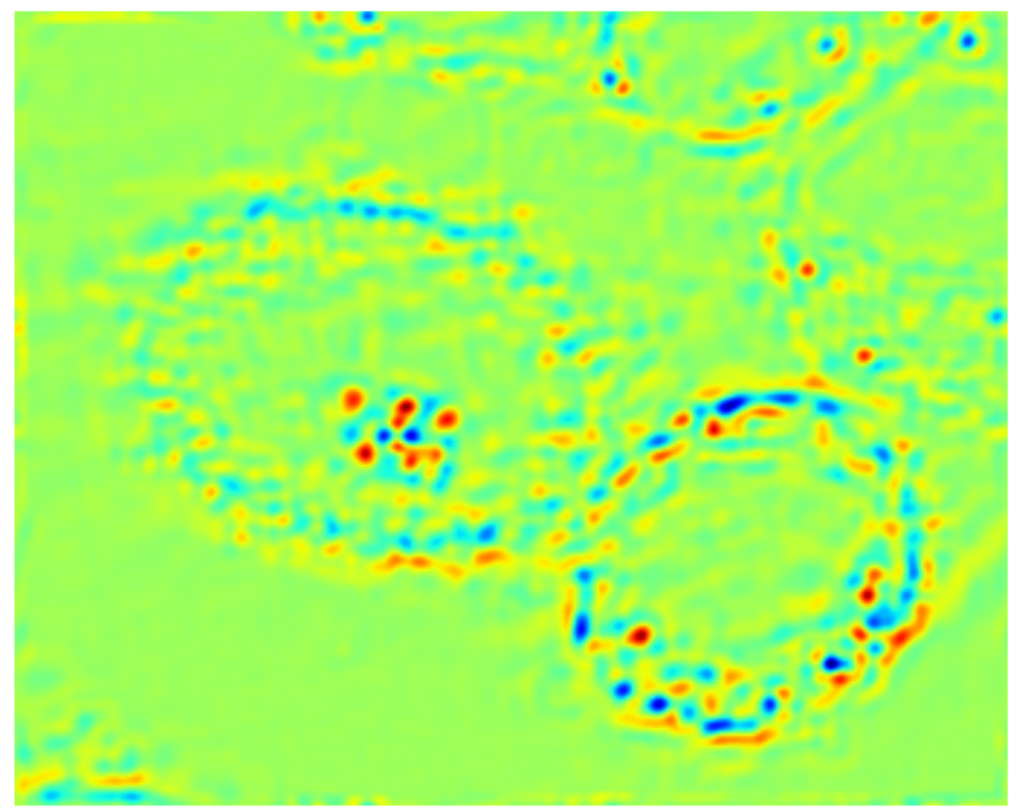

Figure 4: The computed cross-correlation of the Cell and Spot images, computed via explicit convolution. 

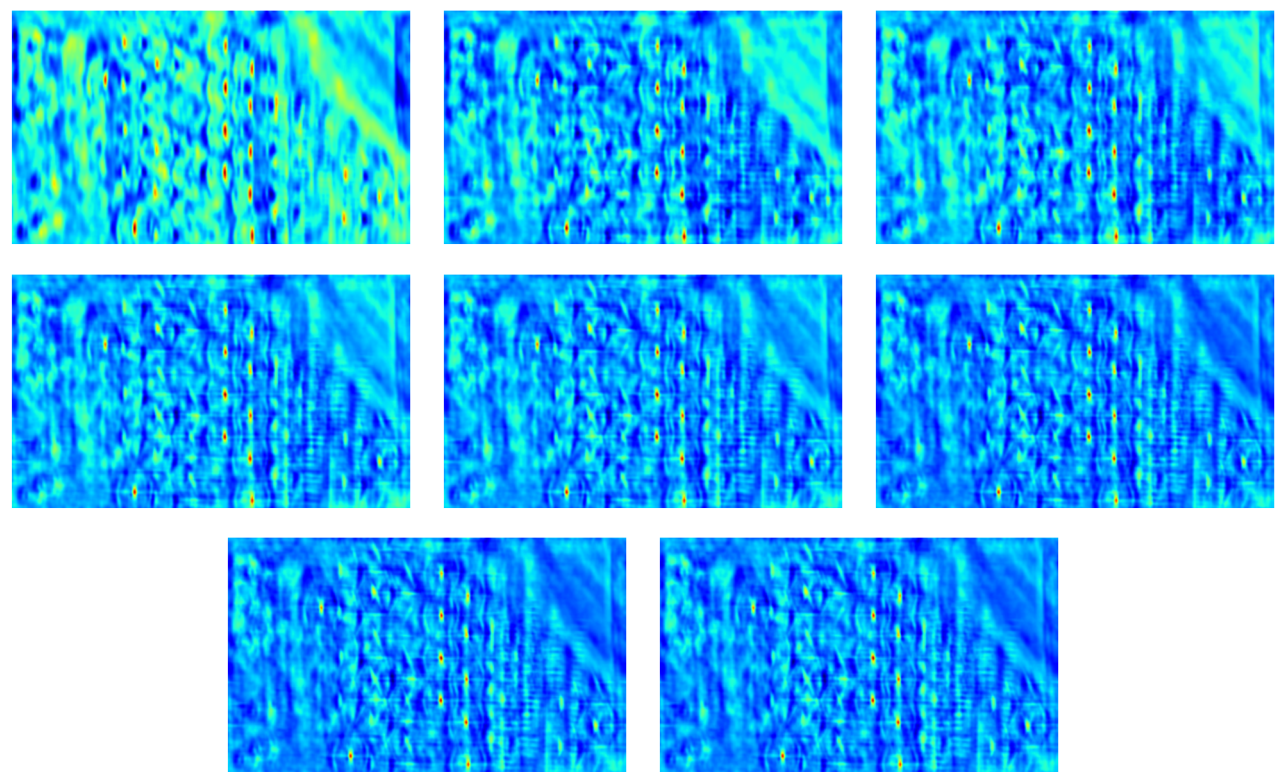

Figure 5: Approximations of the cross-correlation of the Planes and Plane images, computed via explicit convolution. Shown (from left to right, top to bottom) are the estimated cross-correlations from rank 1,2,3,4,5,10,20, and 30 approximations of the Plane image. The rank-2 approximation suffices for detection of all planes oriented like the template.

\begin{tabular}{|c|c|c|}
\hline Type & Running time (s) & Relative error \\
\hline Full image & 1.302 & - \\
Rank-1 & $9.038 \cdot 10^{-3}$ & $8.720 \cdot 10^{-1}$ \\
Rank-3 & $2.499 \cdot 10^{-2}$ & $5.831 \cdot 10^{-1}$ \\
Rank-6 & $4.917 \cdot 10^{-2}$ & $8.746 \cdot 10^{-2}$ \\
Rank-9 & $7.278 \cdot 10^{-2}$ & $7.168 \cdot 10^{-3}$ \\
Rank-12 & $9.683 \cdot 10^{-2}$ & $2.018 \cdot 10^{-3}$ \\
Rank-15 & 0.1215 & $1.669 \cdot 10^{-4}$ \\
Rank-18 & 0.1446 & $1.995 \cdot 10^{-4}$ \\
Rank-21 & 0.1683 & $3.184 \cdot 10^{-4}$ \\
Rank-24 & 0.1924 & $2.436 \cdot 10^{-4}$ \\
Rank-27 & 0.2167 & $2.291 \cdot 10^{-5}$ \\
Rank-30 & 0.2402 & $7.657 \cdot 10^{-7}$ \\
Rank-33 & 0.2639 & $2.992 \cdot 10^{-7}$ \\
Rank-38 & 0.3054 & $2.560 \cdot 10^{-15}$ \\
\hline
\end{tabular}

Table 1: Tabulation of the running times and relative errors, averaged over five runs, for computed cross-correlations of the Planes and Plane images. Both the direct and the approximated cross-correlations were computed using explicit convolution operations. 

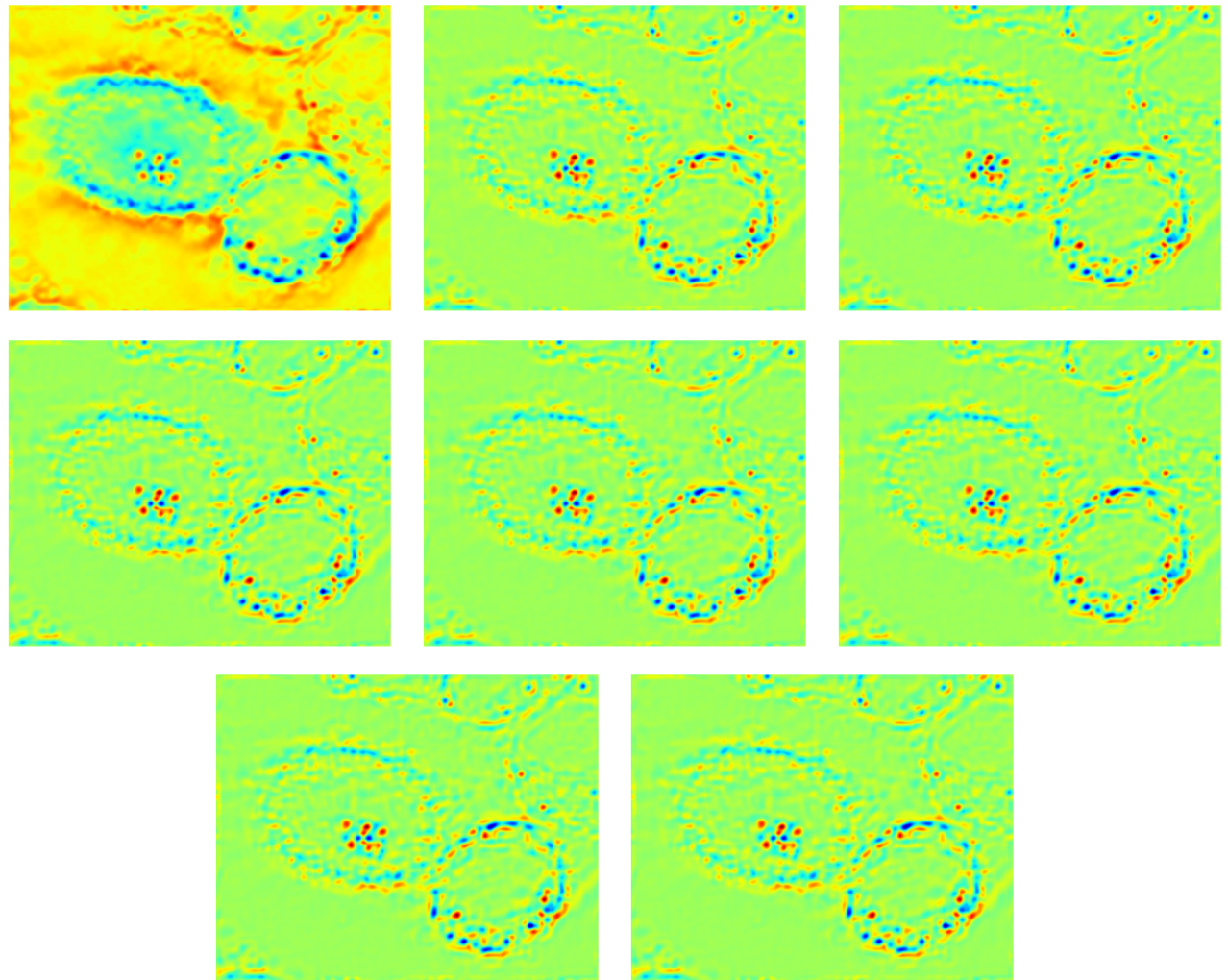

Figure 6: Approximations of the cross-correlation of the Cell and Spot images, computed via explicit convolution. Shown (from left to right, top to bottom) are the estimated cross-correlations from rank 1,2,3,4,5,6, 8, and 11 approximations of the Spot image. The rank-2 approximation suffices for detection of all large spots.

\begin{tabular}{|c|c|c|}
\hline Type & Running time (s) & Relative error \\
\hline Full image & $1.188 \cdot 10^{-1}$ & - \\
Rank-1 & $4.707 \cdot 10^{-3}$ & 1.352 \\
Rank-2 & $7.630 \cdot 10^{-3}$ & $1.043 \cdot 10^{-1}$ \\
Rank-3 & $1.186 \cdot 10^{-2}$ & $1.135 \cdot 10^{-2}$ \\
Rank-4 & $1.368 \cdot 10^{-2}$ & $2.532 \cdot 10^{-3}$ \\
Rank-5 & $1.833 \cdot 10^{-2}$ & $1.378 \cdot 10^{-3}$ \\
Rank-6 & $2.350 \cdot 10^{-2}$ & $7.829 \cdot 10^{-4}$ \\
Rank-7 & $2.323 \cdot 10^{-2}$ & $6.087 \cdot 10^{-4}$ \\
Rank-8 & $2.653 \cdot 10^{-2}$ & $4.212 \cdot 10^{-4}$ \\
Rank-9 & $2.850 \cdot 10^{-2}$ & $2.130 \cdot 10^{-4}$ \\
Rank-10 & $3.380 \cdot 10^{-2}$ & $9.777 \cdot 10^{-5}$ \\
Rank-11 & $3.523 \cdot 10^{-2}$ & $8.134 \cdot 10^{-15}$ \\
\hline
\end{tabular}

Table 2: Tabulation of the running times and relative errors, averaged over five runs, for computed cross-correlations of the Cell and Spot images. Both the direct and the approximated cross-correlations were computed using explicit convolution operations. 

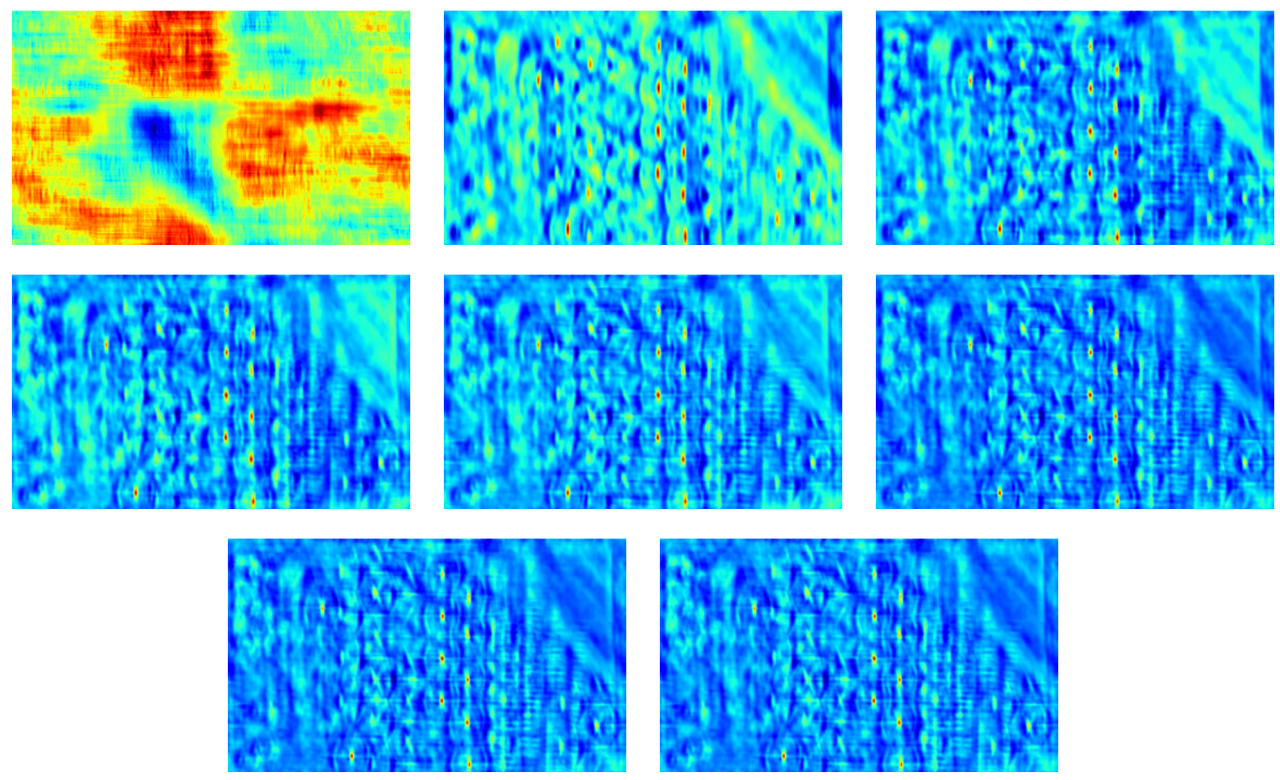

Figure 7: Approximations of the cross-correlation of the Planes and Plane images, computed via FFT-based convolution. Shown (from left to right, top to bottom) are the estimated cross-correlations from rank 1,2,3,4,5,10,20, and 30 approximations of the Plane image. The rank-3 approximation suffices for detection of all planes oriented like the template.

approximation of the Plane image, the computation of the cross-correlation required only $1.92 \%$ of the CPU time needed for the full explicit calculation of the cross-correlation image. The detection of the Spot within the Cell by cross-correlation with a rank-2 approximation of the Spot image, required only $6.42 \%$ of the CPU time demanded for calculating the cross-correlation using the full kernel using the explicit formulas of Algorithm 1.

We turn to FFT-based computations using Algorithm 2. Just as above, we approximate the Plane image by matrices of rank 1, 2, 3, 4, 5, 10, 20, 30 and the Spot image by matrices of rank 1,2,3,4,5,6,8,11. Figures 7 and 8 display the computed results and Tables 3 and 4 show CPU times and approximation errors.

Also when using FFT-based methods, the resulting cross-correlations obtained with approximations of the kernels of very low rank successfully reveal instances of the kernel images within the main images. For both the Plane and Spot kernel images, rank-3 approximations were sufficient for complete detection. The approximated FFT-based cross-correlation between the Planes image and a rank-3 approximation of the Plane kernel was computed in $36.3 \%$ of the CPU time needed for 2D FFT based computations using the full kernel. The corresponding computations for the Cell image and a rank-3 approximation of the Spot kernel required $70.8 \%$ of the time needed for $2 \mathrm{D}$ FFT-based computations with the full kernel. It is notable that the gains realized for the FFT-based computations were far less than those achieved for 

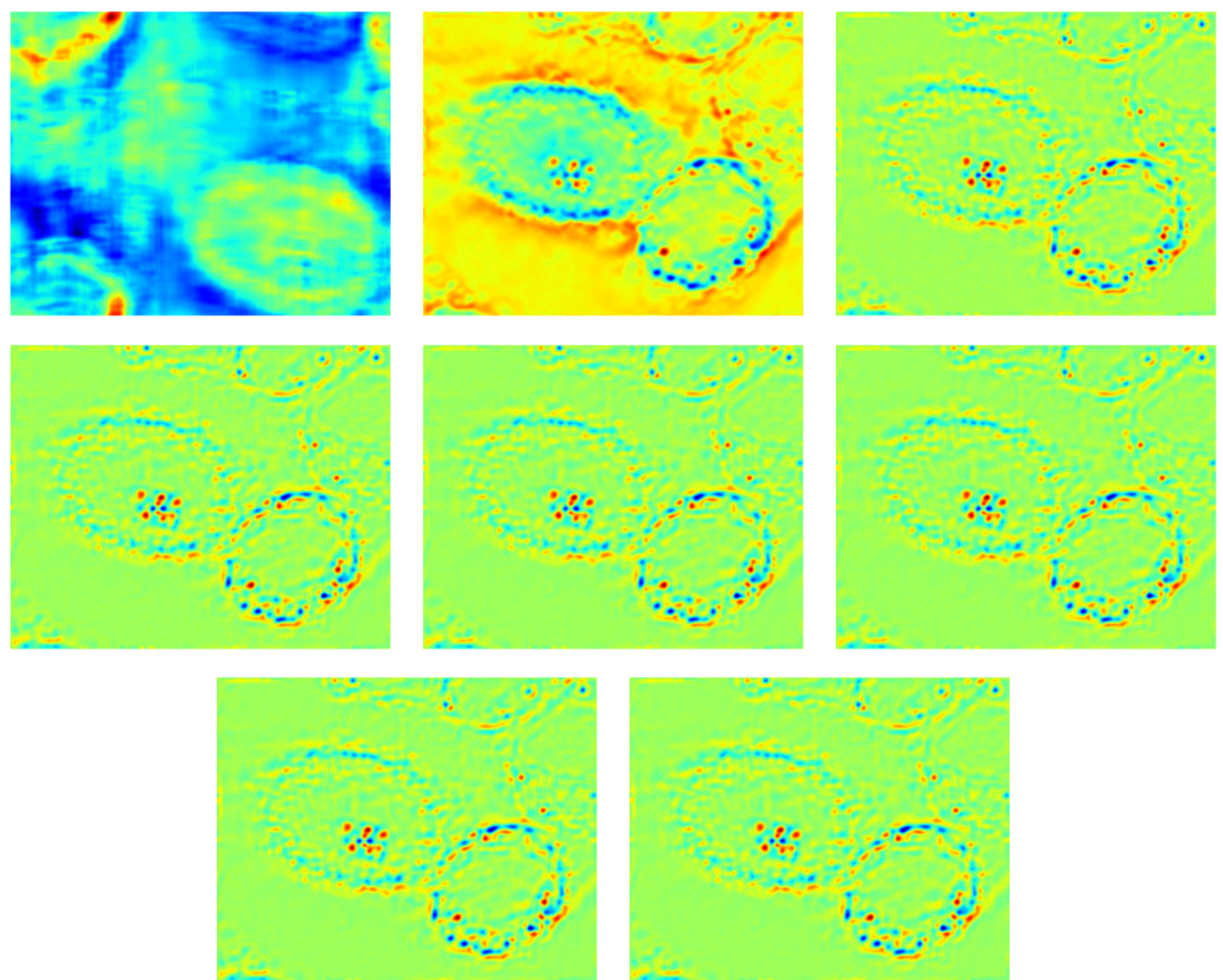

Figure 8: Approximations of the cross-correlation of the Cell and Spot images, computed via FFT- based convolution. Shown (from left to right, top to bottom) are the estimated cross-correlations from rank 1,2,3,4,5,6,8, and 11 approximations of the Cell image. The rank-3 approximation suffices for detection of all large spots.

\begin{tabular}{|c|c|c|}
\hline Type & Running time (s) & Relative error \\
\hline Full image & 0.2072 & - \\
Rank-1 & 0.0720 & $6.393 \cdot 10^{-1}$ \\
Rank-3 & 0.0753 & $5.557 \cdot 10^{-1}$ \\
Rank-6 & 0.0807 & $4.534 \cdot 10^{-1}$ \\
Rank-9 & 0.0794 & $5.538 \cdot 10^{-3}$ \\
Rank-12 & 0.0819 & $8.521 \cdot 10^{-4}$ \\
Rank-15 & 0.0844 & $5.793 \cdot 10^{-4}$ \\
Rank-18 & 0.0873 & $2.507 \cdot 10^{-4}$ \\
Rank-21 & 0.0909 & $2.800 \cdot 10^{-4}$ \\
Rank-24 & 0.0937 & $2.463 \cdot 10^{-4}$ \\
Rank-27 & 0.0967 & $2.805 \cdot 10^{-5}$ \\
Rank-30 & 0.0981 & $1.482 \cdot 10^{-6}$ \\
Rank-33 & 0.1027 & $2.833 \cdot 10^{-7}$ \\
Rank-38 & 0.1067 & $2.998 \cdot 10^{-15}$ \\
\hline
\end{tabular}

Table 3: Tabulation of the running times and relative errors, averaged over five runs, for computed cross-correlations of the Planes and Plane image. Both the direct and the approximated cross-correlations were computed using FFT based convolutions. 


\begin{tabular}{|l|c|c|}
\hline Type & Running time (s) & Relative error \\
\hline Full rank & 0.1813 & - \\
Rank-1 & 0.1238 & $3.857 \cdot 10^{-1}$ \\
Rank-2 & 0.1258 & $3.528 \cdot 10^{-1}$ \\
Rank-3 & 0.1284 & $2.861 \cdot 10^{-2}$ \\
Rank-4 & 0.1305 & $8.834 \cdot 10^{-4}$ \\
Rank-5 & 0.1332 & $3.780 \cdot 10^{-4}$ \\
Rank-6 & 0.1359 & $2.166 \cdot 10^{-5}$ \\
Rank-7 & 0.1362 & $3.759 \cdot 10^{-5}$ \\
Rank-8 & 0.1366 & $1.167 \cdot 10^{-5}$ \\
Rank-9 & 0.1400 & $1.062 \cdot 10^{-5}$ \\
Rank-10 & 0.1420 & $4.087 \cdot 10^{-7}$ \\
Rank-11 & 0.1421 & $4.639 \cdot 10^{-12}$ \\
\hline
\end{tabular}

Table 4: Tabulation of the running times and relative errors, averaged over five runs, for computed cross-correlations of the Cell and Spot images. Both the direct and the approximated cross-correlations were computed using FFT based convolutions.

the explicit computation of the cross-correlations. We conclude that explicit computation of convolutions with a low-rank matrix can be both faster and yield higher accuracy for the same rank than FFT-based methods.

\subsection{Autocorrelation}

This subsection compares the performance of Algorithms 3 and 4. We first explicitly computed the autocorrelations of the Planes and Cell image using the explicit formulas (4). The images of the autocorrelations are shown in Figures 9 and 10. An important application of the autocorrelation of digital images is the identification and characterization of structures indicative of self-similarity within an image. For instance, notice that the airplanes within the Planes image are quite regularly spaced. Consequently, the offcenter maxima within the autocorrelation of the Planes image can be used to quantify the average spacing of the airplanes. If the spatial resolution of the image is known in physical units, then this information can be used to place an upper bound on the size of the airplanes. This bound may be useful when selecting a kernel image for a cross-correlation detection method.

Figure 11 shows approximations of the autocorrelation of the Planes image using approximations of rank 10,20,30,40, 80,150,200, 210 of the image itself. Figure 12 shows analogous approximations for the Cell image. These approximations were computed using explicit convolution (Algorithm 3). The numerical data for these computations, averaged over five runs, are shown in Table 5. We note that for both images the rank-10 approximation is quite accurate both visually and in terms of relative error. All off-center peaks of the autocorrelation are accurately represented in the approximations computed from rank-10 approximations of the images. These approximations 


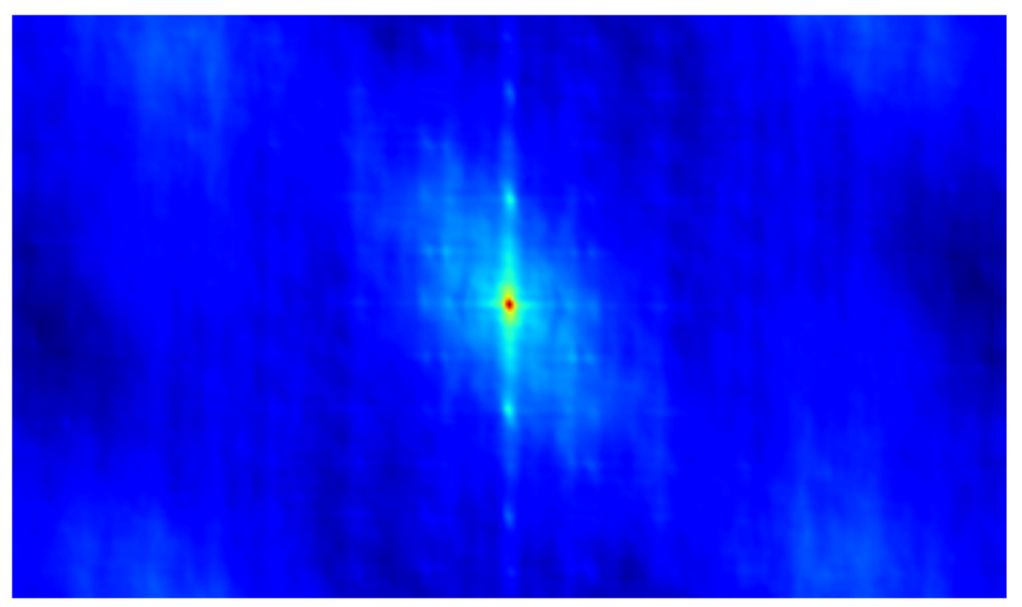

Figure 9: The directly computed autocorrelation of the Planes image, computed via explicit convolution.

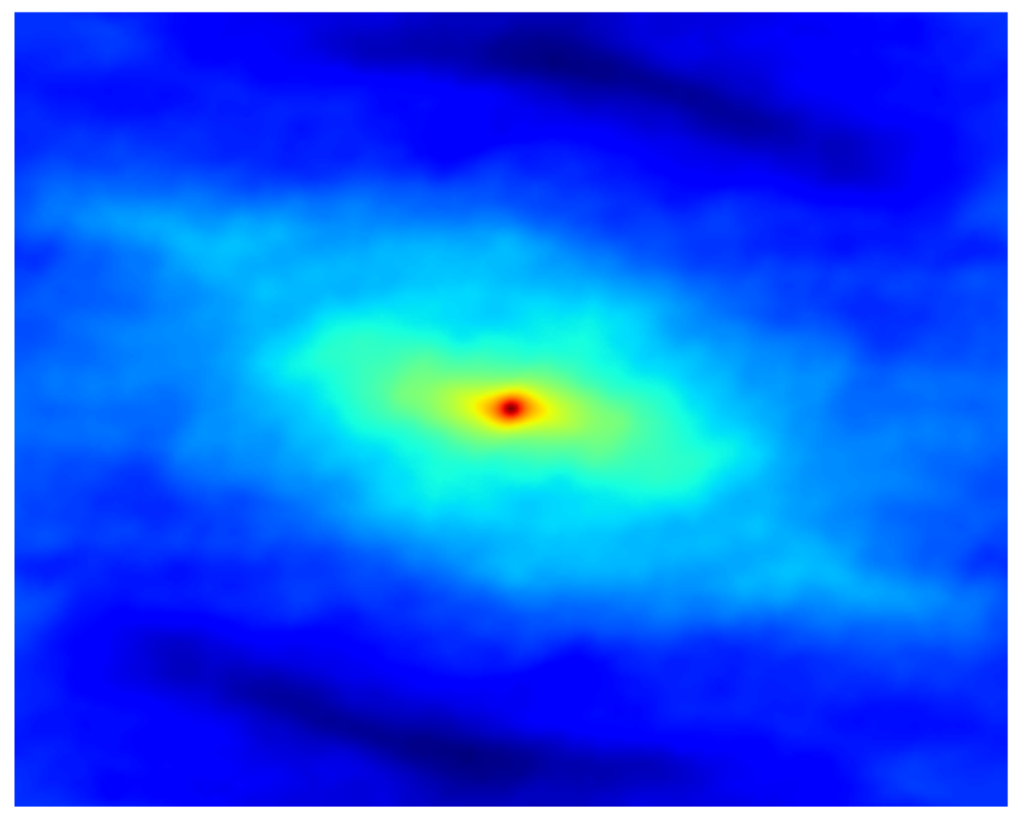

Figure 10: The directly computed autocorrelation of the Cell image, computed via explicit convolution. 


\begin{tabular}{|c|c|c|c|c|}
\hline & \multicolumn{2}{|c|}{ Planes Image } & \multicolumn{2}{c|}{ Cell Image } \\
\hline Type & Running time (s) & Relative error & Running time (s) & Relative error \\
\hline Full image & 62.44 & - & 87.40 & - \\
Rank-10 & 0.03225 & $2.200 \mathrm{e}-05$ & 0.04153 & $8.644 \mathrm{e}-06$ \\
Rank-20 & 0.1245 & $1.280 \mathrm{e}-05$ & 0.1422 & $5.849 \mathrm{e}-06$ \\
Rank-30 & 0.2680 & $6.058 \mathrm{e}-06$ & 0.3074 & $1.657 \mathrm{e}-06$ \\
Rank-40 & 0.4720 & $2.420 \mathrm{e}-06$ & 0.5361 & $5.915 \mathrm{e}-07$ \\
Rank-50 & 0.7324 & $7.255 \mathrm{e}-07$ & 0.8454 & $1.381 \mathrm{e}-07$ \\
Rank-60 & 1.049 & $5.976 \mathrm{e}-07$ & 1.209 & $6.0295 \mathrm{e}-09$ \\
Rank-70 & 1.416 & $4.199 \mathrm{e}-07$ & 1.618 & $1.578 \mathrm{e}-07$ \\
Rank-80 & 1.850 & $2.893-07$ & 2.105 & $1.574 \mathrm{e}-07$ \\
Rank-90 & 2.338 & $1.695 \mathrm{e}-07$ & 2.648 & $6.859 \mathrm{e}-08$ \\
Rank-100 & 2.872 & $8.489 \mathrm{e}-08$ & 3.267 & $2.642 \mathrm{e}-08$ \\
Rank-110 & 3.480 & $6.467 \mathrm{e}-08$ & 3.948 & $2.190 \mathrm{e}-09$ \\
Rank-120 & 4.134 & $9.484 \mathrm{e}-09$ & 4.696 & $5.976 \mathrm{e}-09$ \\
Rank-130 & 4.871 & $5.326 \mathrm{e}-09$ & 5.530 & $7.808 \mathrm{e}-09$ \\
Rank-140 & 5.616 & $3.405 \mathrm{e}-09$ & 6.414 & $9.091 \mathrm{e}-09$ \\
Rank-150 & 6.460 & $2.308 \mathrm{e}-09$ & 7.397 & $6.188 \mathrm{e}-09$ \\
Rank-160 & 7.326 & $1.457 \mathrm{e}-09$ & 8.375 & $1.016 \mathrm{e}-09$ \\
Rank-170 & 8.290 & $1.176 \mathrm{e}-09$ & 9.584 & $9.012 \mathrm{e}-09$ \\
Rank-180 & 9.300 & $7.413 \mathrm{e}-10$ & 10.56 & $1.652 \mathrm{e}-08$ \\
Rank-190 & 10.32 & $4.746 \mathrm{e}-10$ & 11.68 & $2.015 \mathrm{e}-08$ \\
Rank-200 & 11.46 & $3.257 \mathrm{e}-10$ & 12.95 & $1.076 \mathrm{e}-08$ \\
Rank-210 & 12.64 & $1.713 \mathrm{e}-10$ & 14.34 & $5.726 \mathrm{e}-09$ \\
Rank-220 & & & 15.70 & $4.435 \mathrm{e}-09$ \\
Rank-230 & & & 17.11 & $2.751 \mathrm{e}-09$ \\
Rank-240 & & & 18.69 & $2.7140 \mathrm{e}-09$ \\
Rank-250 & & & 20.26 & $5.224 \mathrm{e}-10$ \\
Rank-260 & & & & $3.390 \mathrm{e}-16$ \\
\hline
\end{tabular}

Table 5: Tabulation of the running times and relative errors, averaged over five runs, for computed autocorrelations of the Planes and Cell images. Both the direct and the approximated autocorrelations were computed using explicit convolutions. 


\begin{tabular}{|c|c|c|c|c|}
\hline & \multicolumn{2}{|c|}{ Planes Image } & \multicolumn{2}{c|}{ Cell Image } \\
\hline Type & Running time (s) & Relative error & Running time (s) & Relative error \\
\hline Full image & 0.1414 & - & 0.3087 & - \\
Rank-10 & 0.01373 & 0.0002422 & 0.1529 & 0.0002076 \\
Rank-20 & 0.02407 & 0.0001047 & 0.1594 & $8.245 \mathrm{e}-05$ \\
Rank-30 & 0.03345 & $6.499 \mathrm{e}-05$ & 0.1741 & $3.911 \mathrm{e}-05$ \\
Rank-40 & 0.04417 & $4.4341 \mathrm{e}-05$ & 0.1740 & $2.194 \mathrm{e}-05$ \\
Rank-50 & 0.05347 & $3.052 \mathrm{e}-05$ & 0.1802 & $1.283 \mathrm{e}-05$ \\
Rank-60 & 0.06253 & $2.001 \mathrm{e}-05$ & 0.1966 & $7.608 \mathrm{e}-06$ \\
Rank-70 & 0.06753 & $1.377 \mathrm{e}-05$ & 0.2570 & $4.446 \mathrm{e}-06$ \\
Rank-80 & 0.07999 & $9.836 \mathrm{e}-06$ & 0.2055 & $2.583 \mathrm{e}-06$ \\
Rank-90 & 0.08927 & $6.831 \mathrm{e}-06$ & 0.2149 & $1.573 \mathrm{e}-06$ \\
Rank-100 & 0.1002 & $4.702 \mathrm{e}-06$ & 0.2221 & $9.917 \mathrm{e}-07$ \\
Rank-110 & 0.1082 & $3.446 \mathrm{e}-06$ & 0.2732 & $7.448 \mathrm{e}-07$ \\
Rank-120 & 0.1180 & $2.529 \mathrm{e}-06$ & 0.2782 & $6.263 \mathrm{e}-07$ \\
Rank-130 & 0.1285 & $1.822 \mathrm{e}-06$ & 0.3031 & $5.378 \mathrm{e}-07$ \\
Rank-140 & 0.1348 & $1.270 \mathrm{e}-06$ & 0.3151 & $4.652 \mathrm{e}-07$ \\
Rank-150 & 0.1470 & $8.842 \mathrm{e}-07$ & 0.3647 & $3.982 \mathrm{e}-07$ \\
Rank-160 & 0.1571 & $5.821 \mathrm{e}-07$ & 0.3866 & $3.4071 \mathrm{e}-07$ \\
Rank-170 & 0.1675 & $3.606 \mathrm{e}-07$ & 0.3889 & $2.938 \mathrm{e}-07$ \\
Rank-180 & 0.1760 & $2.078 \mathrm{e}-07$ & 0.4099 & $2.543 \mathrm{e}-07$ \\
Rank-190 & 0.1875 & $1.040 \mathrm{e}-07$ & 0.4283 & $2.113 \mathrm{e}-07$ \\
Rank-200 & 0.1946 & $3.943 \mathrm{e}-08$ & 0.4559 & $1.741 \mathrm{e}-07$ \\
Rank-210 & 0.2007 & $1.320 \mathrm{e}-08$ & 0.4889 & $1.412 \mathrm{e}-07$ \\
Rank-220 & & & 0.3737 & $1.110 \mathrm{e}-07$ \\
Rank-230 & & & 0.3564 & $7.882 \mathrm{e}-08$ \\
Rank-240 & & & 0.3571 & $5.062 \mathrm{e}-08$ \\
Rank-250 & & & 0.3545 & $2.409 \mathrm{e}-08$ \\
Rank-260 & & & 0.4337 & $3.426 \mathrm{e}-20$ \\
\hline
\end{tabular}

Table 6: Tabulation of the running times and relative errors, averaged over five runs, for computed autocorrelations of the Planes and Cell images. Both the direct and the approximated autocorrelations were computed using FFT based convolutions. 

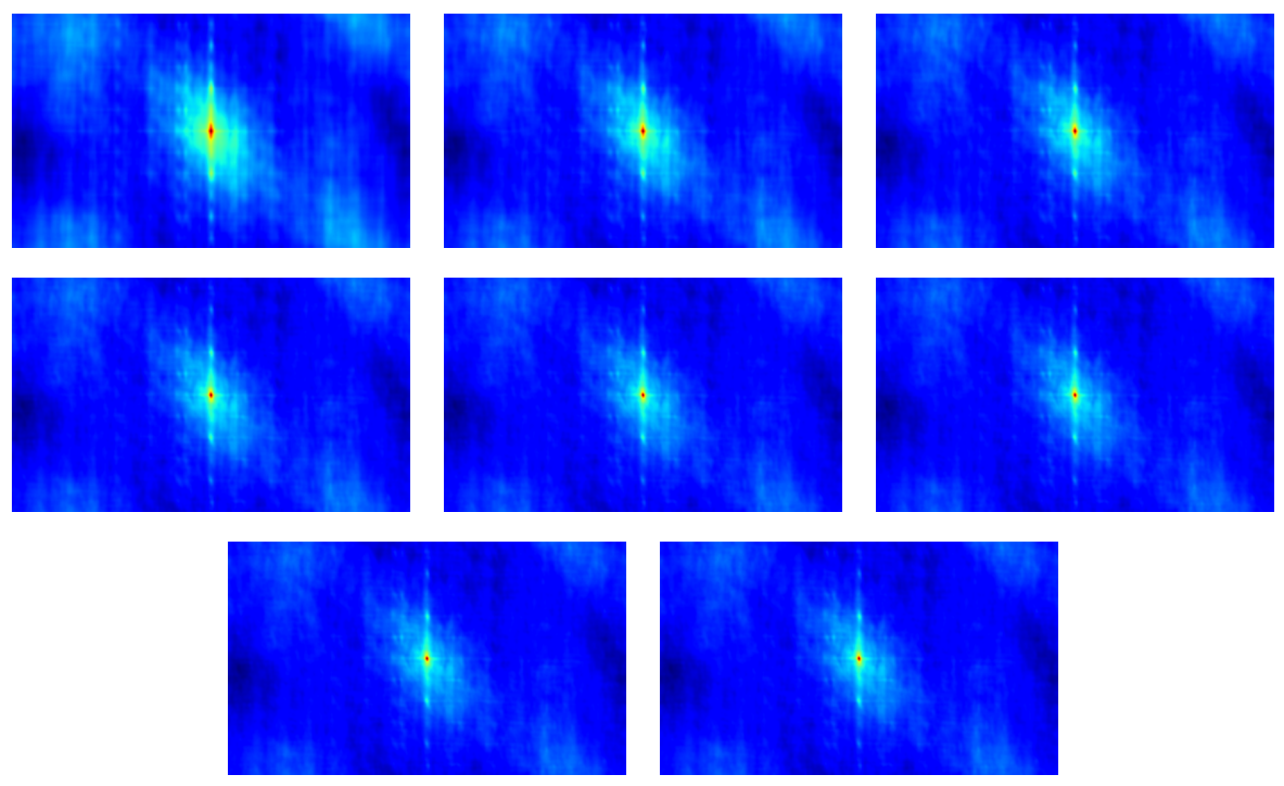

Figure 11: Approximations of the autocorrelation of the Planes image, computed via explicit convolution. Shown (from left to right, top to bottom) are the estimated autocorrelations from rank 10,20,30,40, 80, 150, 200, and 210 approximations of the Planes image. The rank-10 matrix furnishes an accurate approximation.

were computed in far less time than the full explicit computations of the autocorrelations; specifically, in $0.051 \%$ of the time for the Planes image and in $0.047 \%$ of the time for the Cell image.

Figures 13 and 14 show similar approximations of the autocorrelation of the Planes and Cell images, using FFT-based convolutions (Algorithm 4). The numerical data for these computations, averaged over five runs, are tabulated in Table 6 . Just as in the case for the approximation of cross-correlation matrices, we see that the gains realized by the FFT-based algorithm are less than those achieved by explicit convolution. However, the approximations are still accurate enough at low-rank for gains to be realizable.

\section{Discussion and Conclusion}

Both our analysis and computed examples demonstrate that the use of lowrank approximations in convolutive operations can give significant speed-ups when compared with using general matrices. The gains may be realized in application both when computing convolutions explicitly or with the aid of the FFT. Explicit computation is particularly attractive when the convolution is not cyclic, because it yields higher accuracy and may require less time. The examples illustrate that for image processing, results with sufficient accuracy may be achieved with approximations of very low rank. We remark that the relative performance of the methods depends on the properties of 

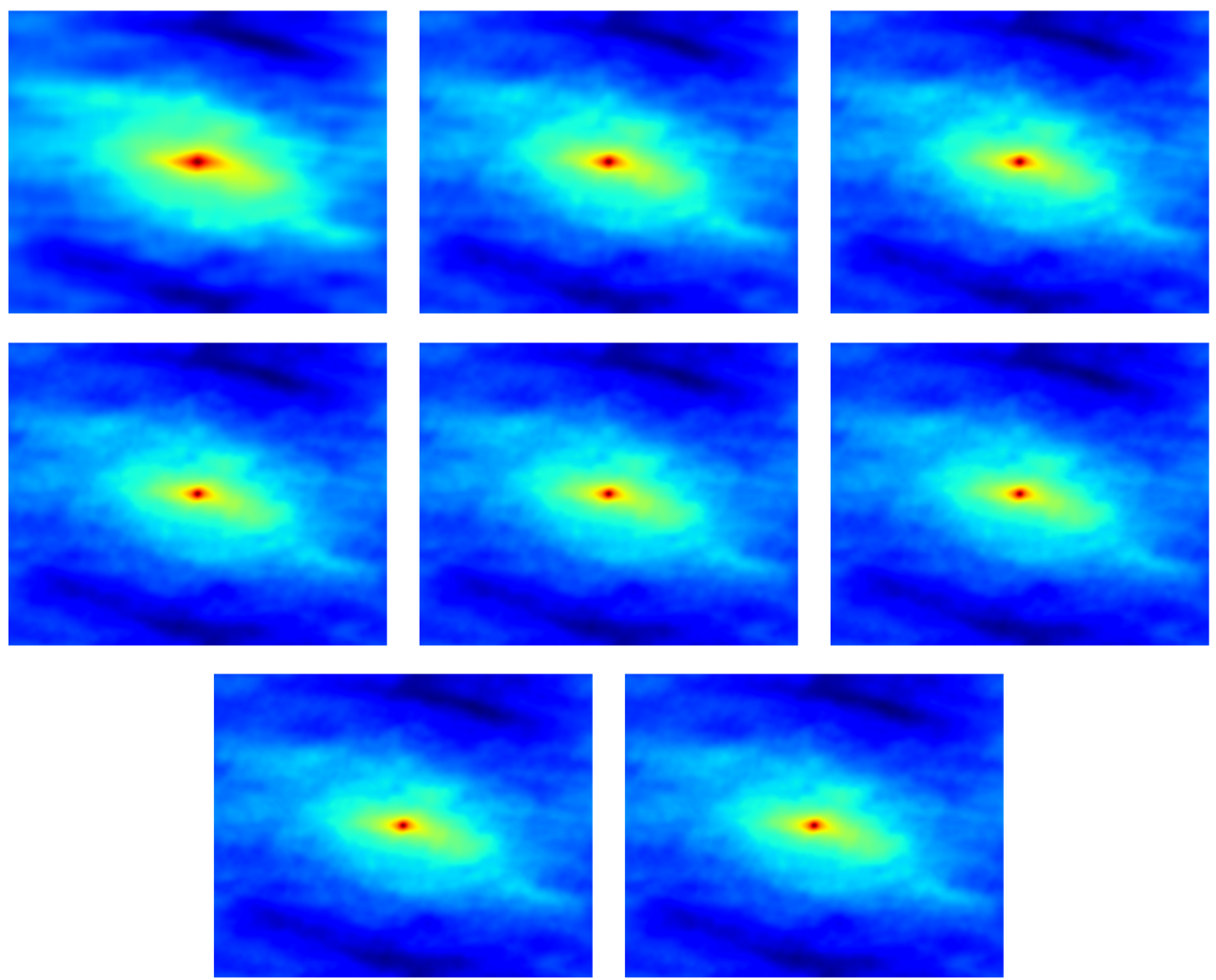

Figure 12: Approximations of the autocorrelation of the Cell image, computed via explicit convolution. Shown (from left to right, top to bottom) are the estimated autocorrelations from rank 10,20,30,50,100,200,220, and 260 approximations of the Cell image. The rank-10 matrix furnishes an accurate approximation. 

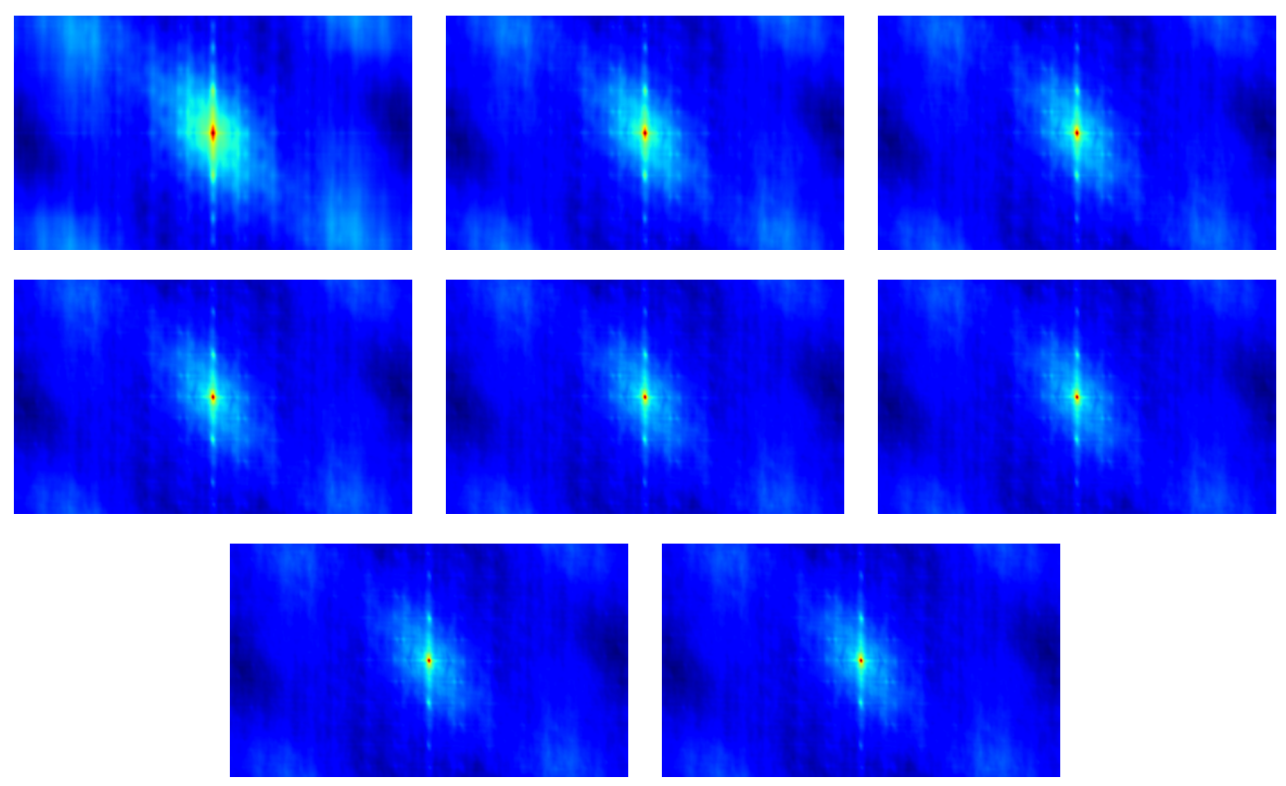

Figure 13: Approximations of the autocorrelation of the Planes image, computed via FFT-based convolution. Shown (from left to right, top to bottom) are the estimated autocorrelations from rank 10, 20,30,40, 80, 150, 200, and 210 approximations of the Planes image. The rank-10 matrix furnishes a sufficiently accurate approximation. It is less accurate than the rank-10 approximation obtained by explicit convolution, but it is faster to compute.

the computer. The overlap-add and overlap-save techniques for implementing FFT-based convolutions may result in speed-ups, see, e.g., Borgering [4] and references therein. These techniques are particularly attractive to use when the desired convolution is circular.

\section{References}

[1] R. Agarwal and J. Cooley, New algorithms for digital convolution, IEEE Trans. Acoustics, Speech and Signal Process., 25 (1977), pp. 392-410.

[2] J. Baglama and L. Reichel, Restarted block Lanczos bidiagonalization methods, Numer. Algorithms, 43 (2006), pp. 251-272.

[3] J. Baglama and L. Reichel, An implicitly restarted block Lanczos bidiagonalization method using Leja shifts, BIT Numer. Math., 53 (2013), pp. 285-310.

[4] M. Borgerding, Turning overlap-Save into a multiband mixing, downsampling filter bank, IEEE Signal Processing Magazine, March 2006, pp. $158-161$. 

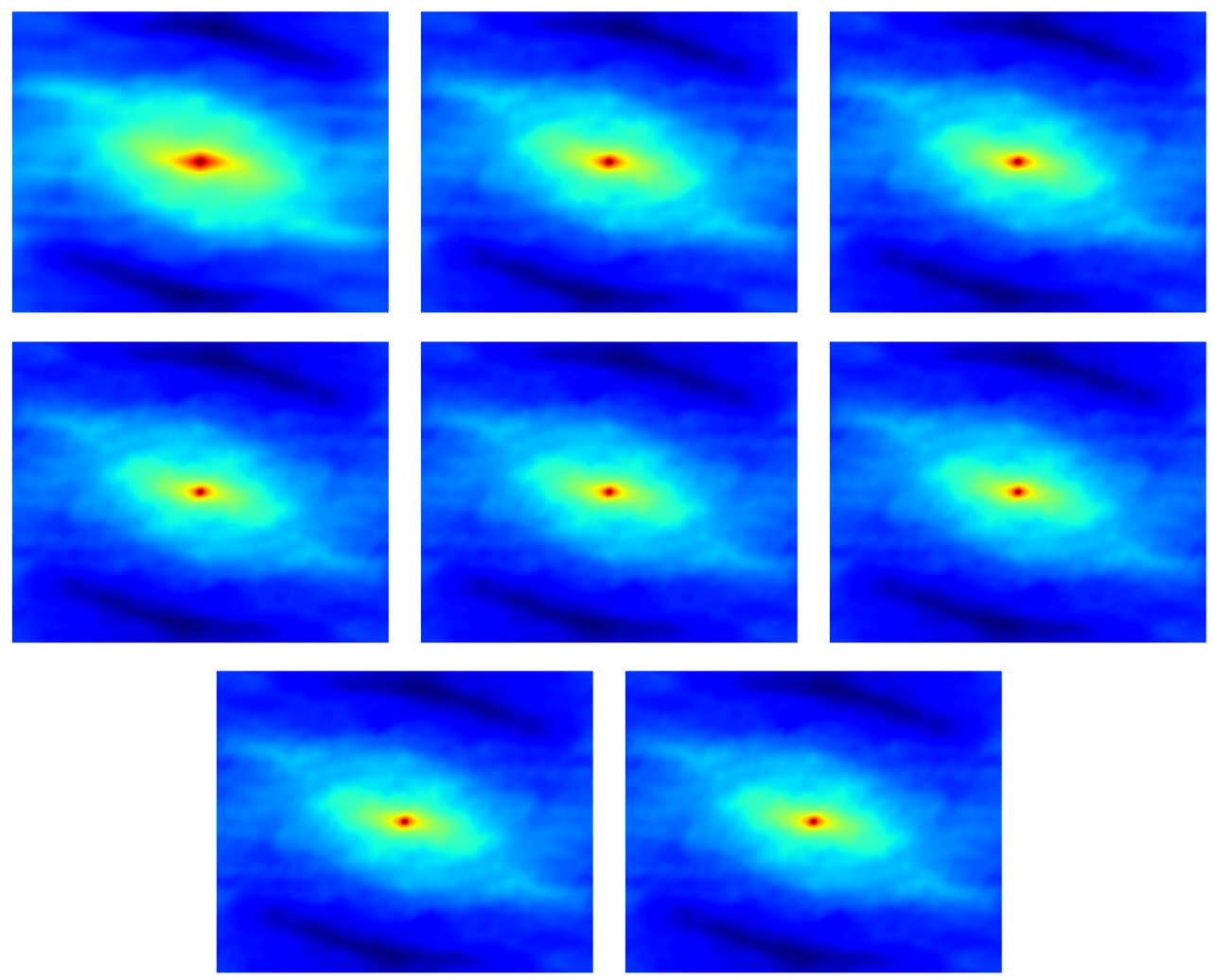

Figure 14: Approximations of the autocorrelation of the Cell image, computed via FFT-based convolution. Shown (from left to right, top to bottom) are the estimated autocorrelations from rank 10,20,30, 50, 100,200,220, and 260 approximations of the Cell image. The rank-10 matrix furnishes a sufficiently accurate approximation. It is less accurate than the rank-10 approximation obtained by explicit convolution, but it is faster to compute. 
[5] R. Brunelli and T. Poggio, Face recognition: Features versus templates, IEEE Trans. Pattern Analysis and Machine Intelligence, 15 (1993), pp. $1042-1052$.

[6] D. Bugajewski and T. Diagana, Almost automorphy of the convolution operator and applications to differential and functional differential equations, Nonlinear Studies, 13 (2006), pp. 129-140.

[7] M. Collins and N. Duffy, Convolution kernels for natural language, Adv. Neural Information Process. Systems, 1 (2002), pp. 625-632.

[8] J. W. Cooley, P. A. W. Lewis, and P. D. Welch, The application of the fast Fourier transform algorithm to the computation of spectra and cross-spectra, J. Sound Vib., 12 (1970), pp. 339-352.

[9] Z. Ding and L. Qiu, Blind MIMO channel identification from second order statistics using rank deficient channel convolution matrix, IEEE Transactions on Signal Processing, 51 (2003), pp. 535-544.

[10] B. Feng and G. Wei, A comparison of the spectral and the discrete singular convolution schemes for the KdV-type equations, J. Comput. Applied Math., 145 (2002), pp. 183-188.

[11] O. Fialka and M. Cadik, FFT and convolution performance in image filtering on GPU, in IV '06: Proceedings of the conference on Information Visualization, IEEE Computer Society, Washington, D.C., 2006, pp. 609-614.

[12] A. Fotheringham, C. Brunsdon, and M. Charlton, Quantitative geography: Perspectives on spatial data analysis, SAGE, 2000.

[13] L. Goldfischer, Autocorrelation function and power spectral density of laser-produced speckle patterns, J. Opt. Soc. Am., 55 (1965), pp. 247252.

[14] R. C. Gonzalez and R. E. Woods, Digital Image Processing (third edition), Prentice Hall, 2008.

[15] A. Graham, Kronecker Products and Matrix Calculus with Applications, Halsted Press, Wiley, New York, 1981.

[16] P. C. Hansen, J. Nagy, and D. P. O'Leary, Deblurring Images: Matrices, Spectra, and Filtering, SIAM, Philadelphia, 2006.

[17] G. H. Hardy, J. E. Littlewood, and G. Pólya, Inequalities, 2nd ed., Cambridge University Press, Cambridge, 1992.

[18] M. Hopf and T. Ertl, Accelerating 3D convolution using graphics hardware (case study), Proc. IEEE Conf. Visualization, 1999, pp. 471-474. 
[19] R. A. Horn and C. R. Johnson, Topics in Matrix Analysis, Cambridge University Press, Cambridge, 1991.

[20] Z. J. Hou and G. W. Wei, A new approach to edge detection, Pattern Recognition, 35 (2002), pp. 1559-1570.

[21] A. Hyvärinen, Independent Component Analysis, Adaptive and Learning Systems for Signal Processing, Communications and Control Series, Wiley-Blackwell, 2001.

[22] J. Kamm and J. Nagy, Kronecker product and SVD approximations in image restoration, Linear Algebra Appl., 284 (1998), pp. 177-192.

[23] D. Kelley and R. Luebbers, Piecewise linear recursive convolution for dispersive media using FDTD, IEEE Trans. Antennas and Propogation, 44 (1996), pp. 792-797.

[24] T. Kreis, Frequency analysis of digital holography with reconstruction by convolution, Optical Engineering, 41 (2002), pp. 1829-1839.

[25] D. Krubsack and R. Niederjohn, An autocorrelation pitch detector and voicing decision with confidence measures developed for noise- corrupted speech, IEEE Trans. Signal Process., 39 (1991), pp. 319-329.

[26] T. Mackie, J. Scrimger, and J. Battista, A convolution method of calculating dose for 150MV X rays, Medical Physics, 12 (1985), pp. 188-196.

[27] S. Mitra and J. Kaiser, Handbook for Digital Signal Processing, Wiley, 1993.

[28] D. P. O'Leary, Some algorithms for approximating convolutions, Computer Vision, Graphics, and Image Processing, 41 (1988), pp. 333-345.

[29] M. Rudnaya, R. Mattheij, and J. Maubach, Evaluating sharpness functions for automated scanning electron microscopy, J. Microscopy, 240 (2010), pp. 38-49.

[30] M. Rudnaya, R. Mattheij, J. Maubach, and H. ter Morsche, Autocorrelation-based sharpness functions, Proc, 3rd IEEE International Conference on Signal Processing Systems, Yantai, China, 2011.

[31] R. Steinbauer and J. Vickers, The use of generalized functions and distributions in general relativity, Classical and Quantum Gravity, 23 (2006), pp. $91-114$.

[32] G. Wei, Solving quantum eigenvalue problems by discrete singular convolution, Physics B, 33 (2000), pp. 343-352.

[33] S. Wong, M. Jasiunas, and D. Kearney, Fast 2D convolution using reconfigurable computing, Proceedings of the Eighth International Symposium on Signal Processing and Its Applications, 2005, pp. 791-794. 